\section{LA-UR-97 $=5186$}

Title: $\quad$ Synopsis of Moisture Monitoring by Neutron Probe in the Unsaturated Zone at Area G

Author(s): $\quad$ Erik Vold *

Environmental Management Program

Los Alamos National Laboratory

* currently at X-HM

EM-SWO

Submitted to:
PEORTED

APR 051838

OSTI

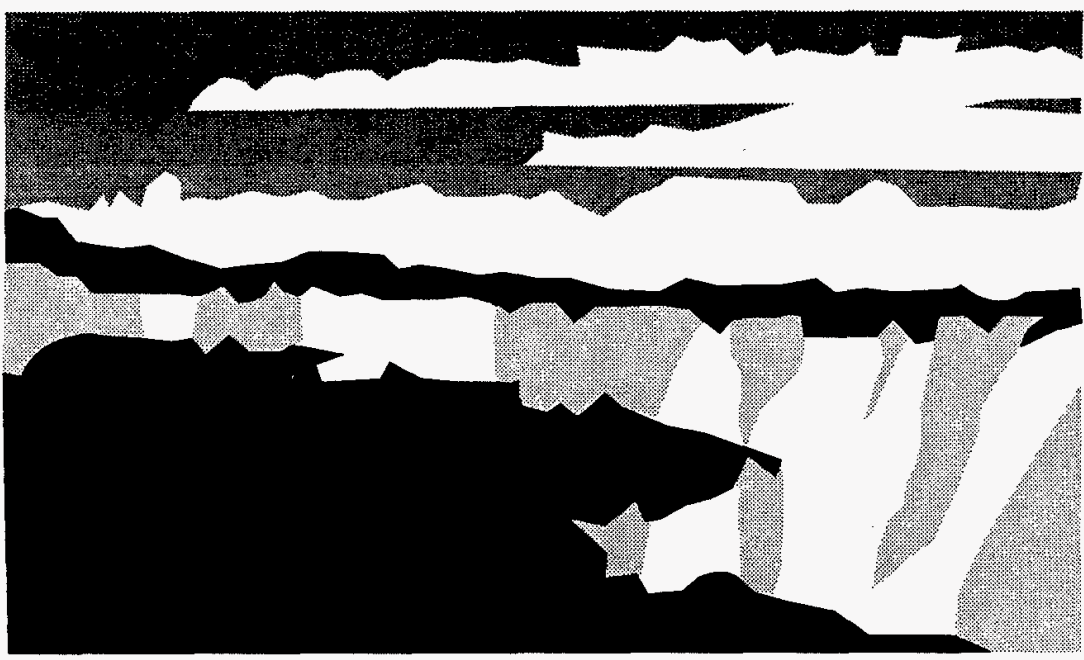

Los Alamos National Laboratory, an affirmative action/equal opportunity employer, is operated by the University of California for the U.S. Department of Energy under contract W-7405-ENG-36. By acceptance of this article, the publisher recognizes that the U.S. Government retains a nonexclusive, royalty-free license to publish or reproduce the published form of this contribution, or to allow others to do so, for U.S. Government purposes. The Los Alamos National Laboratory requests that the publisher identify this article as work performed under the auspices of the U.S. Department of Energy. 


\section{DISCLAIMER}

This. report was prepared as an account of work sponsored by an agency of the United States Government. Neither the United States Government nor any agency thereof, nor any of their employees, makes any warranty, express or implied, or assumes any legal liability or responsibility for the accuracy, completeness, or usefulness of any information, apparatus, product, or process disclosed, or represents that its use would not infringe privately owned rights. Reference herein to any specific commercial product, process, or service by trade name, trademark, manufacturer, or otherwise does not necessarily constitute or imply its endorsement, recommendation, or favoring by the United States Government or any agency thereof. The views and opinions of authors expressed herein do not necessarily state or reflect those of the United States Government or any agency thereof. 


\begin{abstract}
Moisture profiles from neutron probe data provide valuable information in site characterization and to supplement ground water monitoring efforts. The neutron probe precision error (reproducibility) is found to be about $0.2 \mathrm{vol} \%$ under in-situ field conditions where the slope in moisture content with depth is varying slowly. This error is about 2 times larger near moisture spikes (e.g., at the vapor phase notch), due to the sensitivity of the probe response to vertical position errors on the order of 0.5". Calibrations were performed to correct the downhole probe response to the volumetric moisture content determined on core samples. Calibration is sensitive to borehole diameter and casing type, requiring 3 separate calibration relations for the boreholes surveyed here. Power law fits were used for calibration in this study to assure moisture content results greater than zero.
\end{abstract}

Findings in the boreholes reported here confirm the broad features seen previously in moisture profiles at Area G, a near-surface region with large moisture variability, a very dry region at greater depths, and a moisture spike at the vapor phase notch (VPN). This feature is located near the interface between the vitrified and devitrified stratigraphic units and near the base of the mesa. Comparing borehole profiles from several locations, moisture content is qualitatively observed to decrease as one approaches the mesa edge, showing a trend consistent with drying of the mesa interior from the mesa edge.

Borehole moisture shows significant seasonal variations in time at near-surface locations with maximum contents in the autumn following the summer showers and in the spring corresponding with the time of snow melt. These distinct variations (several percent volumetric content) are restricted to the uppermost depths in the borehole profiles, less than 10' only. Below this depth, the moisture profiles are approximately constant in time, however, some variations at these depths can be discerned as statistically significant by appropriately averaging over spatial intervals and considering trends over long times ( 1 year). Specifically, moisture profiles appear to be impacted by disposal operations at least at one location (1121) and probably at a second borehole (1107) with moisture increases seen over the study period. These increases correspond to an average increase of hydraulic conductivity (estimate for the recharge rate under unit gradient conditions) of several $\mathrm{mm} / \mathrm{yr}$ to $1-2 \mathrm{~cm} / \mathrm{yr}$ at the $50-60$ ' depth interval in 1107 . In pit 37 , a moisture pulse associated with a 'bath-tubbing' effect due to grading operations near the moisture monitor port was observed which is large compared to the nominal recharge rates expected at Area G. Moisture profiles at the drier conditions near the mesa edge (1117) do not appear to be significantly influenced by disposal operations.

Continued and improved moisture monitoring programs are recommended to assure the actual site performance conforms to the assumptions made in the Performance Assessment analyses in the wake of continuing impact from ongoing disposal operations. 


\title{
Synopsis of Moisture Monitoring by Neutron Probe in the Unsaturated Zone at Area G
}

\author{
Erik Vold
}

\section{Contents:}

\section{Introduction}

\section{Methods}

Calibration Procedure

Calibration Results

Calibration: borehole 1107

Calibration: borehole 1117

Calibration: borehole 1121

Calibration: 3 hole (4.5" diameter)

Calibration: G-5 (6" diameter holes)

\section{Uncertainty}

Sensitivity of Calibration to Profile Variations in Time

Error Analysis for the In-field Neutron Probe Moisture Measurements

\section{Results}

Baseline Moisture Profiles

Time Dependent Variations

\section{Conclusions}

\section{Acknowledgments}

\section{References}

\section{Figures}




\section{Introduction}

Los Alamos National Laboratory operates a low level radioactive disposal facility at Area G. Environmental protection and assessment of both the operational and post-closure performance of the disposal facility are assured by comprehensive on-going environmental and in-situ monitoring programs. The in-situ monitoring program provides data for site characterization in the Performance Assessment [Hollis, et.al., 1997], required by the U.S Department of Energy for the site operation. The in-situ monitoring program also compliments environmental surveillance of the groundwater pathway in the semi-arid region where a significant unsaturated zone $(>250 \mathrm{~m})$ precludes the effectiveness of monitoring the groundwater directly.

Moisture monitoring of the near surface unsaturated zone is an important element of the on-going in-situ monitoring program which includes radioanalytic analyses and moisture content measurements. Analytic results have been reported previously in special studies (e.g., Puglisi and Vold,1995). The unsaturated zone moisture content and its variability in space and time is critical to understand the recharge rate, the long term performance of the site for retaining contaminants during prolonged leaching, and transport from the disposal site into the unsaturated zone and eventually to the groundwater. Moisture monitoring throughout the near surface unsaturated zone has been reported [Loaiza and Vold 1995] and interpreted as estimates of the local recharge rate [Vold,1996]. Moisture data was combined in that study with hydraulic properties of the subsurface strata to estimate the moisture flux or recharge rate critical to understanding the potential environmental impact of the site.

This report describes the in-field calibration methods used for the neutron moisture probe measurements and summarizes preliminary results of the monitoring program in the in-situ monitoring network at Area $G$. Reported results include three main areas: calibration studies, profiles from each of the vertical boreholes at Area G, and time dependent variations (monthly profiles over a year duration) in a select subset of boreholes. Results are reported here for the vertical borehole network. Results from the horizontal borehole network will be described when available.

\section{Methods}

In-situ moisture content can be measured by a variety of methods [Stephens, 96] [Wilson,et.al.,95]. The availability of several vertical boreholes at Area $G$ allows the use of neutron moisture probes which offer a simple and quick measurement method with excellent measurement precision. The probe is a Campbell Pacific Nuclear [CPN Company, Martinez, CA] Model, CPN 503DR, with a $50 \mathrm{mCi} 241 \mathrm{Am} / \mathrm{Be}$ neutron source. The probe emits high energy neutrons and counts the low energy neutron backscatter. Backscatter is primarily from the hydrogen atoms in the the soil and thus the counts are 
proportional to the water content. The counts are sensitive to borehole diameter and to the casing material if it is hydrogenous (e.g., PVC pipe). The backscatter volume is estimated by the manufacturer to be approximately a sphere with radius about 6 " beyond the open borehole for typical conditions, depending upon moisture content. The probe response function is expected to be an exponential decay with increasing radius from the probe.

The probe is placed down hole with each location measured from the ground surface $(z=0)$. Probe counts are accumulated for a $16 \mathrm{~s}$ interval which gives in the range of 500 to 5000 counts proportional to the local moisture content. At moisture contents $>5 \%$ there are at least 1000 counts per count interval. The error associated with the random count process is $\sim \sqrt{\mathrm{N}} / \mathrm{N}$, or for $N>1000$ counts, the precision error is $<3 \%$. The absolute error is dependent upon the accuracy in the probe calibration procedure. Data is recorded as raw counts per 16s interval at each depth and a standard count is recorded each day to assure consistent comparisons between profiles on different days. It is found that the standard count is very nearly constant and constitutes a negligible correction to the raw data. The results reported here were not corrected for the standard count. This may contribute to a small variability of the data over time as discussed in detail later.

Calibration Procedure

The location of the boreholes measured in this report are shown in Fig.1. The boreholes drilled for the Environmental Restoration Project in 1995 are $4.5^{\prime \prime}$ diameter. These are calibrated against core sample moisture content data from boreholes 1107, 1117 and 1121. Core samples from the boreholes were collected by the Environmental Restoration Project and analyzed for moisture content at the time of borehole drilling [Krier, Marin, Kisiel, pers.com,1996]. These weight percent measurements are converted to volume per cent using stratigraphic unit averaged values for bulk density from [Krier, et.al.,95] except as discussed in the following results. These holes are all cased down to $10^{\prime}$ only to allow the holes to be sealed when not in use for monitoring. The steel casing seems to have a minimal impact on the results, but has not been treated explicitly in the calibrations, and therefore increases the uncertainty of the moisture results in the first 10' depth.

The boreholes 1110, 1111 and G-5 are 6" diameter and these are calibrated to core sample moisture content data from G-5, measured analytically as volume percent for each core sample [DBSA, 95]. These boreholes are uncased. The vertical boreholes in pit $37(37 \mathrm{w}=$ pit west end and $37 \mathrm{c}=$ pit central location) are 2.5 " aluminum cased pipes, which allows a direct moisture volumetric content readout of the neutron probe from the manufacturer's calibration. These cased boreholes were not independently calibrated to field data.

The probes are calibrated by first collecting raw counts throughout a borehole profile at 1 ' intervals. The raw count readings which correspond to the depth locations of the core sample moisture content data are then paired with that moisture content data (typically, at least 10 data points per borehole) 
and a correlation regression is applied to the two data sets. This provides a direct calibration of the probe raw count readings to the measured in-situ moisture content. The standard correlation coefficient, $r^{2}$, provides a measure of the goodness of fit, a rough indicator of the accuracy of the calibration. Since the core sample data and the neutron probe profiles are taken at different times, there is a possibility that moisture content has changed between the two measurements. This is likely to be a small effect based on the data discussed in the following Calibration Results.

\section{Calibration Results}

The resulting moisture content volume percent profiles in the four boreholes used in the calibration are shown in Fig. 2 for reference. These profiles are determined from the final calibrations ( 3 hole - power law fit for boreholes 1107, 1117, 1121, and G-5 power law fit for G-5). Calibration of each of the holes is first discussed to address several issues of concern in deriving these calibrations. The calibrations for the similar diameter holes are then combined to provide a single calibration curve for the $4.5^{\prime \prime}$ holes (from the 3 holes: 1107, 1117, 1121) and for the 6" holes (from G-5).

An important issue is the form of the calibration fit where we have compared linear fits and power law fits. Linear fits are the industry standard, but have the disadvantage that moisture content results can be less than zero in very dry regions under certain calibrations. The power law fit has the advantage that it guarrantees a positive moisture content for any raw count value. A detailed analysis of the probe response function was not done but it would be expected to show that theoretically a power law fit is more accurate to describe the exponential backscattering of the neutrons in approximately spherical geometry.

Calibration: borehole 1107

The calibration curve for data from borehole 1107 alone is shown in Fig.3, as a power law fit to the data. The power law (PL) fit for moisture content, $\theta$, to neutron probe (raw counts $/ 16 \mathrm{~s}$ ), $R_{\mathrm{p}}$, is:

$$
\theta[P L-1107]=4.846 e-4 R_{p}{ }^{1.246}
$$

with correlation coefficient, $r^{2}=0.82$, indicating a good correlation. A linear fit (LF) to the same data gives:

$$
\theta[L F-1107]=3.057 e-3 R_{p}-0.843
$$

with correlation coefficient, $r^{2}=0.79$, indicating a good correlation but slightly less than the power law fit. In this case, the linear fit gives negative moisture content for raw counts below 275 , which did not occur in this profile.

The moisture profile calibrated to this fit is compared to the profile calibrated to the 3-hole power law fit (described later in this section), and to the core sample data in Fig.4. Generally, the core sample data appears to have 
much larger variations, partly attributed to real variations between the smaller sample volumes and partly attributed to the larger errors associated with the moisture determination for each core sample. The two power law fits give very nearly the same result. There appears to be a consistent difference between the neutron data and the core samples with core sample results low in the top $60^{\prime}$ and high in the 60-100' interval. This may be attributed to the bulk density conversion from weight percent to volume percent in the core sample data, if actual bulk density differed consistently from the 'stratigraphic unit averaged values' used in this analysis.

Calibration: borehole 1117

The calibration curve for data from borehole 1117 alone is shown in Fig.5, comparing a power law and a linear fit to the data. The power law (PL) fit for moisture content based on stratigraphic unit averaged bulk density, $\theta\left[\right.$ aveGrow], to neutron probe (raw counts $/ 16 s$ ), $R_{p}$, is:

$$
\theta[\text { PL-1117,aveGrow }]=7.987 e-5 R_{p}{ }^{1.468}
$$

with correlation coefficient, $r^{2}=0.61$. A linear fit (LF) to the same data gives:

$$
\theta[L F-1117 \text {,aveGrow }]=5.005 e-3 R_{p}-2.917
$$

with correlation coefficient, $r^{2}=0.78$. In this case, the linear fit gives a much better correlation but it yields negative moisture content for raw counts below 583 , which occured at one location in this profile.

The assumed values for bulk density appeared to be important in this calibration as also seen for the previous borehole, 1107. To examine the sensitivity of the calibration to the bulk density, we took values of bulk density determined experimentally in borehole G-5 [DBSA,1995] and projected these values onto borehole 1117 , while normalizing to the depths of the major stratigraphic unit interfaces. This was intended to provide another estimate of the local bulk density used to calculate volume percent from the core moisture data. Using these core sample data (vol\%-core-G5row), another set of calibrations were obtained for the neutron probe as:

$$
\theta[\text { PL-1117, G5row }]=5.289 e-5 R_{p}^{1.524}
$$

with correlation coefficient, $r^{2}=0.61$. A linear fit $(\mathrm{LF})$ to the same data gives:

$$
\theta[L F-1117, \text { G5row }]=4.882 e-3 R_{p}-2.823
$$

with correlation coefficient, $r^{2}=0.79$. The power law fit is sensitive to this change in the bulk density correction to the volumetric percent while the linear fit is fairly insensitive. The correlations remain unchanged. 
The moisture probe profile results (for the first 1117 calibration in Eqn.3) are compared to the two core sample volumetric content results (based on the two conversions of the core data to the volumetric results as described above) in Fig. 6. The two estimates of bulk density provide very similar estimates of volumetric moisture content from the weight percent data. The core data still appears to have a larger local variation than in the neutron probe profile.

Calibration: borehole 1121

The calibration curve for data from borehole 1121 alone is shown in Fig.7, as a power law fit to the data. The power law (PL) fit for moisture content, $\theta$, to neutron probe (raw counts $/ 16 \mathrm{~s}$ ), $R_{\mathrm{p}}$, is:

$$
\theta[P L-1121]=1.0286 e-3 R_{p}^{1.139}
$$

with correlation coefficient, $r^{2}=0.59$. A linear fit $(\mathrm{LF})$ to the same data gives:

$$
\theta[L F-1121]=2.859 e-3 R_{p}+0.267
$$

with correlation coefficient, $r^{2}=0.57$, indicating a weak correlation, slightly less than the power law fit.

The moisture profile calibrated to each of these fits are compared in Fig.8. The results agree very precisely at lower moisture contents and diverge slightly with increasing moisture content to a maximum difference between the estimates for the moisture contents in this profile of about $5-7 \%$ (relative error). In Fig.9, the moisture profile calibrated to the power law fit is compared to the core sample data. As seen previously, the core sample data appears to have much larger variations than the neutron probe profile.

Calibration: 3 hole (4.5" diameter)

As seen above, the calibrations for each hole differ slightly depending upon the distribution of moisture data in that particular borehole. Practically, it is desirable to have a single calibration for all holes of one diameter $\left(4.5^{\prime \prime}\right.$, in this case) and casing type (none, in this case). The calibration data from the 3 holes (3H): 1107, 1117, and 1121 are combined into a single plot in Fig.10. The power law (PL) fit for moisture content, $\theta$, to neutron probe (raw counts/16s), $\mathrm{R}_{\mathrm{p}}$, is:

$$
\theta[P L-3 H]=1.6776 e-4 R_{p}{ }^{1.3764}
$$

with correlation coefficient, $r^{2}=0.81$. A linear fit (LF) to the same data gives:

$$
\theta[L F-3 H]=3.9736 e-3 R_{p}-1.66069
$$


with correlation coefficient, $r^{2}=0.79$, indicating a good correlation but slightly less than the power law fit. The correlations to either calibration fit are better than in any single hole indicating the fit is improved by spanning the larger range in moisture content throughout the three holes.

These power law fit calibration values provide the basis for all subsequent neutron probe moisture profiles in $4.5^{\prime \prime}$ diameter boreholes discussed throughout this report. The power law fit was selected over the linear fit to avoid negative moisture results in dry regions of the borehole.

Calibration: G-5 (6" diameter holes)

All 6" borehole profile results are from the calibration in G-5. Volumetric moisture content was determined experimentally [DBSA, 95] on 10 samples at approximately $10^{\prime}$ intervals throughout the hole. These data are presented for calibration with the corresponding neutron probe readings in Fig. 11. The power law (PL) fit for moisture content, $\theta$, to neutron probe (raw counts/16s), $R_{p}$, is

$$
\theta[P L-G 5]=4.00543 e-4 R_{p}^{1.3161}
$$

with correlation coefficient, $r^{2}=0.91$. A linear fit (LF) to the same data gives

$$
\theta[\mathrm{LF}-1121]=5.9267 e-3 R_{p}-2.5646
$$

with correlation coefficient, $r^{2}=0.91$, indicating a good correlation in both cases. The good correlation coefficients may in part be attributed to the fact that the core sample data was directly evaluated in volumetric content and no average bulk density values were needed to estimate the volumetric core sample moisture content.

In either fit, the leading coefficient is greater for the 6 " hole than for the smaller $4.5^{\prime \prime}$ hole. This indicates there is a significantly smaller return of scattered neutrons in the larger hole at a given moisture content in the surrounding matrix. This effect is seen in Fig. 12 which compares the moisture profile from the appropriate G-5 (6" diameter) calibration to the resulting profile if one applied the $3 \mathrm{H}\left(4.5^{\prime \prime}\right)$ calibration from the wrong diameter borehole. This clearly indicates the strong sensitivity of the calibration coefficients to the borehole diameter.

\section{Uncertainty}

Sensitivity of Calibration to Profile Variations in Time

The calibrated moisture profile is compared to the volumetric core sample data for borehole G-5 in Fig. 13. There is close agreement overall, but there is an apparent discrepency at the $42^{\prime}$ and $52^{\prime}$ samples which appear to be significantly lower than the neutron probe profile values. This may be related to real changes in the profile over this depth interval which occured in the uncased hole between the core sample results (collected in late 1994) 
and the time of these neutron probe measurements (mid 1996). Fig. 14 again compares the core sample volumetric results with the recent (1996) neutron moisture probe profile and also compares a neutron moisture profile which was taken in 1994 shortly after the drilling [from Loaiza and Vold 1995]. That profile was calibrated in 1994 to the same G-5 core sample data in a similar method as used here. The two neutron probe results are in close agreement throughout the profile except in the interval from about $40^{\prime}$ to $60^{\prime}$. This further indicates that a real change in this interval of the profile has occured in the two years since drilling. This introduces additional uncertainty into the G-5 calibration but the good agreeement in profiles outside of the $40^{\prime}$ to 60 ' interval suggests the influence of the time variation of the profile on the calibration is very small.

Variation in the profile between time of core sampling and neutron moisture probe measurements is also an issue of concern in the calibration of the ER Project holes (4.5" diameter boreholes drilled in late 1995). To address this concern, profiles were taken at several intervals over time in one borehole, 1117, starting only two days after drilling was complete $(11 / 29 / 95)$. Moisture differences were calculated at every depth between succussive profile measurements as shown in Fig. 15, which includes the absolute moisture profile at the initial time for reference. The profile measurements were made at times (with respect to the drilling completion) of 2 days, 9 days, 94 days, 147 days and 247 days. At 9 days and at 94 days, two profiles were made with the differences between profiles on the same day representing a measure of the overall measurement error associated with reproducility under in-field conditions. (Note that this series of measurements were made before the field procedure was 'standardized' to collect neutron probe data in raw counts, so the calibration of these data differed slightly from the standard calibration used for the other results reported in this study.)

The time differenced moisture profile data is shown in Fig.16 and in Fig. 17 which allows different perspectives on the same data set. The variations throughout the profiles at different dates are similar to the variations between the profiles taken on the same days (at 9 days and at 94 days) and indicates that the field error associated with reproducability dominates and masks any real profile trends in time. Averages and standard deviations of the data in Fig.16 or 17, are summarized in Table I over the whole borehole profile and over selected depth intervals. These values concur that the same-day variations are comparable to or exceed changes over time in most cases suggesting that there is no significant change in time for this data set (additional data is presented in the section, Time Dependent Variations).

This important result indicates that the procedure for calibration of neutron probe profiles against core samples taken at earlier times are reasonably accurate, within the uncertainty associated with the precision of the in-field measurements. Qualitatively in Fig.17 and quantitatively in Table I, we see that a standard deviation of less than about $0.2 \%$ volumetric 
moisture content is a good estimate of the error associated with in-field reproducibility for most times and at most depth intervals.

The largest variation (seen in Fig.16, 17 or Table I) occurs near the moisture spike at $\sim 80^{\prime}$ depth and for the profile taken at $t=247$ days, where the change in moisture content clearly exceeds the differences between the same day measurements. This may indicate a real change, however, in Fig.16 or 17, it appears that the local error 'overshoots' at a depth above the moisture spike and then 'undershoots' at a depth just below the spike. This suggests an error in vertical positioning such that the moisture spike is determined to be at a slightly different depth on this profile. The error at this location is large because it is sensitive to the rapid variation of moisture content with depth in the steady profile. This is discussed further in the following section on Error Analysis. The problem of trying to distinquish a real trend in time from the field data variation is quantified in greater detail in a later section, Time Dependent Variations.

\section{Table I}

Average and standard deviation of the change (delta values) in moisture content (vol\%) from the borehole moisture profile or profile segment at the indicated time compared to the moisture data at the previous time for Borehole 1117.

\begin{tabular}{|c|c|c|c|c|c|c|}
\hline \multicolumn{6}{|l|}{ delta valu } & \\
\hline ave & 0.011548 & -0.11976 & 0.122739 & 0.030545 & 0.042279 & 0.003166 \\
\hline stdev & 0.1846 & 0.187806 & 0.195271 & 0.289446 & 0.170026 & 0.168902 \\
\hline \multicolumn{7}{|c|}{ data $10^{\prime}-30^{\prime}$} \\
\hline ave & 0.005676 & -0.08372 & 0.114943 & 0.028381 & 0.038314 & -0.02483 \\
\hline stdev & 0.219059 & 0.187259 & 0.170352 & 0.237041 & 0.161414 & 0.165204 \\
\hline \multicolumn{7}{|c|}{ data $30^{\prime}-50^{\prime}$} \\
\hline ave & 0.01788 & -0.08568 & 0.073755 & 0.02682 & 0.01043 & 0.009685 \\
\hline stdev & 0.177791 & 0.155213 & 0.202025 & 0.160522 & 0.145195 & 0.154488 \\
\hline \multicolumn{7}{|c|}{ data $50^{\prime}-70^{\prime}$} \\
\hline le & 0.052895 & -0.1125 & 0.12516 & -0.00671 & 0.00745 & 0.018625 \\
\hline dev & 0.140564 & 0.132344 & 0.156003 & 0.208683 & 0.139146 & 0.143069 \\
\hline \multicolumn{7}{|c|}{ data $70^{\prime}-90^{\prime}$} \\
\hline e & -0.03215 & -0.20311 & 0.180368 & 0.076068 & 0.116847 & 0.010979 \\
\hline dev & 0.194986 & 0.248443 & 0.245129 & 0.477857 & 0.216507 & 0.216312 \\
\hline
\end{tabular}


Error Analysis for the In-field Neutron Probe Moisture Measurements

The neutron probe error is related to the statistical counting error, to variations in time due to instrument drift and to the error associated with repositioning the probe at a specific borehole location. This last error will contain contributions from the error in radial location within the borehole (if the borehole diameter exceeds the probe diameter significantly, as it does for most of our boreholes), and from error in the vertical location or depth (z).

The relative counting error for $\mathrm{N}$ neutrons counted in the interval was discussed earlier and is on the order of $\sqrt{\mathrm{N}} / \mathrm{N}$, or about $\sqrt{500 / 500} \sim 0.045$ at the driest locations and $\sqrt{2000} / 2000 \sim 0.022$ at $7 \%$ volumetric content, decreasing at higher moisture contents. Instrument drift is checked with a standard count source in the field before each borehole profile. This shows a very small variation due to instrument drift (typically less than $1-2 \%$ relative error) which has been neglected in this study but could be used in subsequent work to normalize data at different times and further reduce the instrument drift error. The contribution to error from the radial positioning has been shown in field work within our boreholes to be negligible [Newell, personal communication 1997]. The error due to vertical location, $z$, is sensitive to the vertical variation in the actual moisture profile.

An expression for the uncertainty in the neutron probe measurements is based on a simple 'calibration ' equation for the probe. We assume for simplicity in analysis that the probe is calibrated to a linear fit. Previously, we chose to use the power law fit for practical considerations in assuring a positive moisture content throughout the profile, however, it was shown that the agreement between linear and power law fits is very good and so the calibration error analysis is based on a linear calibration fit for simplicity.

The linear calibration equation for the volumetric moisture content, $\theta$, is rewritten,

$$
\theta=m R_{p}[z]+b
$$

where the neutron probe response, $R_{p}$, is found at an actual depth, $z$. The depth at which we think we are measuring, $z_{0}$, is different from the actual depth by our error in measuring depth, $\Delta z=z-z_{0}$. The measured probe response is related to the probe response at the correct depth (neglecting error in the radial positioning which was determined to be negligible) as

$$
R_{p}[z]=R_{p}\left[z_{0}\right]+\frac{\partial R_{p}}{\partial z} \Delta z
$$

Substituting this into the previous expression and rearranging, one obtains

$$
\theta=\left\{m R_{p}\left[z_{0}\right]+b\right\}+\left\{m \frac{\partial R_{p}}{\partial z} \Delta z\right\}
$$


which shows the field result depends on where one thinks they are, $z_{0}$, and where they actually are, $z=z_{0} \pm \Delta z$.

This last expression allows one to evaluate an equation for the error estimate in the reported moisture, $\sigma_{\theta}$, based on the standard propagation of errors [Bevington, 69]. For notation simplicity $R_{p}{ }^{\prime}=\partial R_{p} / \partial z$. The propagation of errors in the moisture content, expressed in terms of the errors in the right hand side terms, $\sigma_{\mathrm{m}}, \sigma_{\mathrm{Rp}}, \sigma_{\mathrm{b}}, \sigma_{\mathrm{Rp}}$ and $\sigma_{\Delta \mathrm{z}}$ is expanded to

$$
\begin{aligned}
\sigma_{\theta}{ }^{2}= & R_{p}{ }^{2} \sigma_{m}{ }^{2}+m^{2} \sigma_{R p}{ }^{2}+\sigma_{b}{ }^{2}+ \\
& \left(\Delta z R_{p}\right)^{\prime}{ }^{2}{\sigma_{m}}^{2}+(m \Delta z)^{2} \sigma_{R p^{\prime}}{ }^{2}+\left(m R_{p}{ }^{\prime}\right)^{2} \sigma_{\Delta z}{ }^{2}
\end{aligned}
$$

where the first 3 terms on the right hand side (RHS) contribute to calibration errors assuming the probe is correctly located and the last 3 terms on the RHS are associated with vertical positioning errors. The first 3 terms on the RHS can be combined into one term for an overall calibration error, $\sigma_{\mathrm{cal}}{ }^{2}$, where $\sigma_{\text {cal }}$ was found in Table I to be $\sim 2 \times 10^{-3}$ in moisture volume percent at most locations. Comparing terms 1 and 4 on the RHS, the term 4 is only significant (or vertical location contributes significantly to the overall calibration error in the estimated slope, $m$ ) if

$$
\Delta z R_{p}^{\prime}>R_{p}
$$

Data in borehole 1117 (Fig.15-17 and Table I) near a depth of 80' shows a maximum slope in the moisture profile, $R_{p}{ }^{\prime}[\max ]$, occurs near the vapor phase notch corresponding to about $4 \%$ volumetric moisture content per foot depth. Using this value for $R_{p}{ }^{\prime}[\max ]$, and values of $R_{p}$ from the linear fits given in the previous calibration sections, then term 4 in Eqn.16 is only significant when $\Delta z$ exceeds about $1.6 \mathrm{ft}$. This implies a vertical positioning error of greater than $1 \mathrm{ft}$, which is unlikely to occur under field conditions, and so term 4 is usually negligible. The slope in probe reponse is known, so term 5 on the RHS of Eqn.16 is negligible.

With these considerations and using the overall calibration error, $\sigma_{\mathrm{cal}}$, then the error in moisture content determined by the probe measurement is written

$$
\sigma_{\theta}{ }^{2} \sim \sigma_{c a l^{2}}+\left(m R_{p}\right)^{2} \sigma_{\Delta z}{ }^{2} .
$$

This implies the error due to probe vertical positioning will be significant when

$$
\sigma_{c a l}<\left(m R_{p}{ }^{\prime}\right) \sigma_{\Delta z}
$$


or when the position error, $\sigma_{\Delta z}$, exceeds a value such that

$$
\sigma_{\Delta z}>\sigma_{c a l} /\left(m R_{p}{ }^{\prime}\right) .
$$

It was noted that the steepest profile observed is about $4 \%$ volumetric moisture content per foot or $\partial \theta / \partial z \sim\left(m R_{p}{ }^{\prime}\right) \sim 4 x 10^{-2}$. The positioning error is greatest at this location. The overall calibration error was seen to be $\sigma_{c a l} \sim 2 \times 10^{-3}$, so the positioning error becomes significant in regions of steep moisture slope (near the VPN) if

$$
\sigma_{\Delta z}>2 \times 10^{-3} / 4 \times 10^{-2}=0.05 \mathrm{ft}=0.6 \mathrm{in} .
$$

This shows that a very small error in the position of the probe, of a magnitude expected for typical variations in in-field conditions, will enhance the overall error in regions where the moisture profile is steep. The data in Table I and the corresponding moisture profiles in Fig.15-17, suggest that the moisture content error, at a location with a small gradient in moisture content, is about $0.18 \%$ (volumetric percent). The error increases where the moisture gradient is significant, and near the Vapor Phase Notch, the error approximately doubles due to the uncertainty in the exact depth location as reflected in Eqn.21.

\section{Results}

Baseline Moisture Profiles

Boreholes drilled by the ER Project for Area G site characterization in 1994 and 1995 were passed to the WM Project for continuing in-situ monitoring in 1996. Based on the 3 hole power law calibration for boreholes of 6" diamater and the G-5 calibration for boreholes of 4.5" diameter, results for the calibration boreholes were shown in Fig.2. Results are shown in Figs. 18 to 20 for a baseline moisture profile from each of the additional boreholes (located in Fig.1) accessed to date.

The three boreholes in Fig.18 are deep enough to include the Vapor Phase Notch. The three profiles in Fig. 19 are from boreholes near the east end of Area $G$ which extend to less than $60^{\prime}$ depth. Four profiles from the central portion of Area $G$ are compared together in Fig. 20. Comparing each of these figures to the borehole locations in Fig.1 it is qualitatively apparent that the moisture profiles are wetter towards the interior of the mesa and drier near the edges of the mesa. This is particularly clear for most depth intervals in the upper 60' of the boreholes central to Area G (Fig.20). A quantitative estimate of this mesa edge drying effect has not yet been done but is possible from these borehole data, e.g., moisture content at a given stratigraphic depth can be plotted verses borehole distance to the nearest mesa rim. 
Time Dependent Variations

Time dependent variations in moisture profiles are important to determine where significant changes to the recharge rate may occur and how the disposal operations may be influencing these changes. The in-situ monitoring program is currently collecting moisture profiles from 7 of the Area $G$ boreholes (1107, 1111, 1117, 1121, G-5, 37w and 37c) on an approximately monthly basis. This data set has been collected since the summer of 1996 (1111 and 37c were added more recently in early 1997). Results of time dependent variations are discussed in this report for the west end of pit $37(37 \mathrm{w})$, and the calibration boreholes, 1107, 1117, and 1121. Data presented here covers almost one year and the time dependent plots shown in this section span the time from August, 1996 to May, 1997.

Moisture profiles in the west end of pit 37 (37w in Fig. 21) show good reproducibility of profile features, evident in the three profiles at top, taken in 1996. The August 1996 profile is in good agreement with the average profile from 1994 (94ave) in the bottom portion of the figure and shows there has been negligible change in the profile for the two year period from 1994 to August, 1996. Moisture profiles in pit 37 (west) taken from August 1996 to May 1997 show a significant pulse of moisture moving through the profile (Fig. 22 and 23). The cause of this pulse has been attributed to a 'bathtubbing' effect in the pit adjacent to the monitoring boreholes, which resulted from a redistribution of the surface topography in the area as part of the on-going disposal operations (this pit is an active disposal pit and is not closed to date). As such, this moisture pulse is not representative of typical conditions. The large moisture pulse has allowed a comparison to detailed transient modeling results leading to an in-field calibration of the computational model. This effort is discussed in a separate report [Vold,1997, in progress].

The difference in moisture content at a given depth between two different times gives a measure of the change or error in the moisture content at that point. A statistically significant slope in moisture content over time is an indication that the change in moisture content is real.

The uncertainty can be reduced by averaging moisture content over a depth interval. We chose to average the profile data in $10^{\prime}$ segments which provides 10 moisture measurements per segment. The error in the segment averaged moisture content is thus reduced from the error associated with each probe measurement by $n / \sqrt{\mathrm{n}}$ or about a factor of 3 when $n=10$ is the number of point measurements averaged in the segment. The profile segment averages are made after taking the time difference (moisture at time, $t$, minus moisture at the previous time or at an initial reference time) at each depth to eliminate the spatially varying profile in moisture content and obtain a measure of only the change (or variation) over subsequent measurements in time.

Plots of moisture content verses depth and time are shown for borehole 1121 in Fig.24. The left figure shows the moisture content at each 
time in volume percent, the center figure shows the moisture difference at each time relative to the initial time profile and the right figure shows the present time moisture content relative to that at the previous time. A seasonal variation is apparent in each case, with obvious change in moisture content in the upper 10'. The two near surface maximum in moisture contents occur in early fall (presumably the moisture pulse following the cumulative summer thunderstorms) and in spring, presumably attributed to the snow melt.

In the right figure and more obvious in the center figure, there is some qualitative indication of changes in moisture which may be occuring down to about 60-70'. In Fig.25 (left side) the time differenced data (from Fig.24-center) is averaged over $10^{\prime}$ intervals to reduce to uncertainty of the avergaed content. Here the seasonal pulses are quite pronounced and the trend to increasing moisture down to $60-70^{\prime}$ is slightly noticable. The same data in Fig.25 (left) is plotted as line plots for the 7 upper 10' depth segments in Fig.25 (right) and for the lower 10' averaged segments in Fig.26. Linear correlations of these moisture contents with the time were calculated for each interval and the correlation statistics are summarized in Table II. The statistics and significance are discussed later.

Similar plots are shown in Fig.27 and 28 for borehole 1117. Figure 27 shows the moisture content, the content relative to the initial profile and relative to the previous profile as a function of depth and time. The data in the central figure was averaged over 10' intervals as previously described and the linear plots verses time are shown in Fig.28 for each of the 10' intervals. Because the VPN straddles two of these segments, an average from $76^{\prime}$ to $85^{\prime}$ is also included to capture the moisture spike at the VPN. There is no apparent change in time for any interval except within 10' of the surface.

The moisture content at each depth verses time is shown for borehole 1107 in Fig.29 in the same 3 plot format as for the previous boreholes. The data in each of these 3 plots are then averaged over the 10' intervals and the results are shown in Fig. 30. This 10' segment averaged data is then plotted as line plots verses time in Fig.31, allowing linear correlations with time to be determined for each interval. The plots show the strong seasonal variation in the upper $10^{\prime}$ interval as previously suggest some moisture changes

Figures 25, 26, 28, and 31 show plots of the 10' segment-averaged time change in moisture content (profile at time, $t$, minus that at the initial time) verses time for each of the intervals in the three calibration boreholes. Linear correlations of these moisture contents with time were calculated for each interval and the correlation statistics are summarized in Table II.

A simple but rigorous quantification of the statistical significance of a trend in time can be made from the linear correlation of moisture in time using the estimated slope, $\mathrm{m}$, the correlation coefficient, $\mathrm{r}^{2}$, and the standard $t$-test statistic, $t_{\alpha / 2}[\alpha, v]$, where $\alpha$ is a specified level of confidence (e.g., $\alpha=0.025$ for the $95 \%$ confidence level in a two-tailed distribution) and $v=n-2$ is the degrees of freedom, and $n$ is the number of data pairs in the linear correlation. A general criteria for determining when a fitted slope, $\mathrm{m}$, is 
statistically different from the actual slope, b, is [p.289, Walpole and Myers, 78] given by

$$
|m| \leq b \pm \frac{t_{\alpha / 2} S}{S_{x x^{1 / 2}}}
$$

where $S_{x x}$ and $S$ are standard estmators of statistical variance. Relations between these estimators and the estimated slope, $\mathrm{m}$, and correlation coefficient, $\mathrm{r}^{2}$, are [Walpole and Myers, 78] given by

$$
\begin{aligned}
& S^{2}=\frac{S_{y y}-m S_{x y}}{v} \\
& m=S_{x y} / S_{x x}
\end{aligned}
$$

and

$$
r^{2}=m^{2}\left(S_{x x} / S_{y y}\right) .
$$

We are interested in determining when the linear trend is real or when the slope is significantly different from zero. This can be expressed by setting $b=0$ in the above criteria (Eqn.22). Considering the equality in Eqn.22, this defines the 'edge' of statistical confidence that a particular slope estimator is significantly different from zero, which is then

$$
m=\frac{t_{\alpha / 2} S}{S_{x x}{ }^{1 / 2}} .
$$

Substituting in the three relations (Eqn.23-25) between the estimators, the criteria for a statistically significant non-zero slope becomes

$$
m=t_{\alpha / 2} / v^{1 / 2}\left(\left(\frac{m}{r}\right)^{2}-m^{2}\right)^{1 / 2} .
$$

This simplifies, eliminating the estimate of the slope, $\mathrm{m}$, and can be rearranged as

$$
r^{2}=\frac{1 .}{\left(1 .+\frac{v^{1 / 2}}{t_{\alpha / 2}}\right)} .
$$

This prescribes the correlation coefficient, $\mathrm{r}^{2}$, where the fitted slope estimate is marginally statistically significant and different from zero at the $t_{\alpha / 2}$ level of confidence and for $v$ degrees of freedom, or equivalently $n=v+2$ data pairs in the correlation. According to this expression, as the number of data pairs (v) increases, a smaller correlation coefficient is significant at a particular level of confidence, and so a significant non-zero slope is indicated at a lower 
correlation coefficient with more data. As the prescribed level of confidence $\left(t_{\alpha}\right)$ increases, a given data set requires a larger correlation coefficient to be marginally significant at that level. Interestingly, the statistical significance does not depend explicitly in this form on the magnitude of the slope estimate, $\mathrm{m}$. It does, of course, depend on the slope estimate implicitly through the $r^{2}$ value itself.

In the moisture content time series examined in this report, there are 10 or 11 data pairs in time for each of the three boreholes, at each of the 10' averaged depth intervals. For 10 or 11 data pairs the t-statistic differs very slightly $(-2 \%)$. From standard tables, the t-statistic for $v=n-2=9$ and for the $95 \%$ confidence level is: $t_{\alpha / 2}[\alpha=0.025, v=9]=2.262$. The $t$-statistic for the $90 \%$ confidence level is: $t_{\alpha / 2}[\alpha=0.05, v=9]=1.833$, and for the $98 \%$ confidence level, $t_{\alpha / 2}[\alpha=0.01, v=9]=2.821$. This indicates the required correlation coefficient is reasonably sensitive to the desired level of confidence. We will assume a $95 \%$ confidence level for results in this study.

The required correlation coefficient for a slope estimate which is significantly different from zero at the $95 \%$ confidence level under the conditions in this study $(v=9)$ is then

$$
r^{2}=\frac{1 .}{\left(1 .+\frac{(v=9)^{1 / 2}}{t_{\alpha / 2}=2.262}\right)}=0.43 .
$$

For each borehole depth interval where the correlation value exceeds 0.43 , a "yes" is indicated in the last column of Table II to show this correlation is significant at the $95 \%$ confidence level. For these intervals, the slope in the table may be considered as a statistically significant change in moisture content.

The statistical results support the qualitative indications seen in Figs.25-30, and quantitative previously uncertain trends. Each of the three boreholes shows a significant increase in moisture over this time period in the upper most $10^{\prime}$ interval. This is related to the clear seasonal variation. A contributing factor may also be that the previous winter was a very dry season leading to record draughts in early 1996 . This might tend to bias the moisture content at early times in our study to unusually dry values and the increasing moisture throughout the study reflects a 'return' to more normal levels.

Beneath the top $10^{\prime}$ interval, trends are significantly more subtle but are statistically significant in a few cases. No trends below $10^{\prime}$ are seen in borehole 1117, which is among the drier holes observed at Area G, consistent with its location near the mesa edge. Borehole 1121 shows the moisture content significantly increasing in every $10^{\prime}$ interval down to $70^{\prime}$, and no significant changes below that depth. The average change in the upper $70^{\prime}$ is slightly less than $0.04 \mathrm{vol} \% /$ month, or about $0.4 \mathrm{vol} \%$ change per year during the study time. This increase indicates a significant change of almost $1 / 2$ vol\% per year. 
This borehole 1121 is located between two recently place asphalt pads and it is likely there is surface water run-off channeled towards this borehole which is in excess of the average run-off at Area G. This suggests the disposal operations are significantly modifying the undisturbed hydrology to at least $70^{\prime}$ depth at this location (and probably at others similarly situated with respect to surface run-off engineering features. A rough average for the moisture content in this $70^{\prime}$ interval has thus changed over the year from about $8 \%$ to $8.5 \%$. This corresponds to an increase in the unsaturated hydraulic conductivity (assuming Unit $2 \mathrm{~b}$ properties) from about $0.3 \mathrm{~cm} / \mathrm{yr}$ to $0.5 \mathrm{~cm} / \mathrm{yr}$, which serves as a rough estimate of the increase in recharge rate (under unit gradient or uniform moisture profile conditions).

Borehole 1107 shows significant correlations beneath the top 10' interval with moisture content decreasing at 11-20' and at 31-40' and moisture increasing at 51-60'. This latter increase is large, nearly the same as seen in the top $10^{\prime}$, and corresponds to about $1.2 \mathrm{vol} \%$ per year increase over the study year. The combined moisture decrease from the two zones is less than this, corresponding to losses of $0.3-0.4 \mathrm{vol} \%$ per year. This may be attributed to a net evaporation from these deeper layers, but the mechanism is not certain.

Borehole 1107 is centrally located at Area $G$ and may represent conditions throughout a large region within Area $G$, as seen by the similarities in moisture profiles (Fig.20) in several boreholes located near here $(1106,1107,1108$, and G-5). Refering to Fig.14, there was an apparent moisture increase from 1994 to 1996 of about $2 \%$ volumetric content restricted to the depth interval from about $40^{\prime}$ to $60^{\prime}$, seen in the nearby borehole, G-5. This corresponds to about $1 \%$ per year increase in this interval which agrees closely with the $\sim 1.2 \%$ per year increase seen in borehole 1107 at 50-60' (Table II). This suggests a common trend throughout this central portion of Area G may be occuring. A $1 \%$ per year increase at $10 \%$ average moisture content corresponds to an unsaturated hydraulic conductivity increase from about 1 to $2 \mathrm{~cm} / \mathrm{yr}$.

A west-to-east cross-section of the features near the borehole 1107 is drawn to scale in Fig.31. Nearby pits and shafts may be significant in the subsurface moisture changes seen in this borehole. However, that possibility does not support the fact that similar profiles are seen at several nearby boreholes, where the disposal operations features are distinctly different than immediately adjacent to 1107 . Thus, the common stratigraphy between these centrally located holes seems to play a dominant role in the moisture profiles, while the impact of disposal operations is uncertain at these locations.' 
Table II. Moisture Trend Analysis: TA-54 Monitoring Boreholes

\begin{tabular}{|c|c|c|c|c|c|}
\hline Borehole & $\begin{array}{l}\text { Depth } \\
\text { Interval } \\
\text { (feet) }\end{array}$ & $\begin{array}{l}\text { Slope } \\
\text { (vol\%/month) }\end{array}$ & $\begin{array}{l}\text { Intercept } \\
\text { (vol\%) }\end{array}$ & $\begin{array}{l}\text { Correlation } \\
\text { Coefficient } \\
\left(\mathrm{r}^{2}\right) \\
\end{array}$ & $\begin{array}{c}\text { Non-zero } \\
\text { slope (95\% } \\
\text { Confidence) } \\
\end{array}$ \\
\hline \multirow[t]{14}{*}{1121} & $1-10$ & 0.2064 & -0.2772 & 0.4942 & yes \\
\hline & $11-20$ & 0.0885 & -0.3222 & 0.5833 & yes \\
\hline & $21-30$ & 0.0454 & -0.0516 & 0.6026 & yes \\
\hline & $31-40$ & 0.0295 & -0.0705 & 0.5068 & yes \\
\hline & $41-50$ & 0.0329 & -0.0466 & 0.4405 & yes \\
\hline & $51-60$ & 0.0373 & -0.0428 & 0.8233 & yes \\
\hline & $61-70$ & 0.0411 & 0.0348 & 0.8824 & yes \\
\hline & $71-80$ & -0.0013 & -0.0508 & 0.0032 & \\
\hline & $81-90$ & 0.0033 & -0.0304 & 0.0655 & \\
\hline & $91-100$ & -0.0014 & -0.0393 & 0.0110 & \\
\hline & $101-110$ & 0.0086 & -0.0120 & 0.2080 & \\
\hline & $111-120$ & -0.0007 & -0.0151 & 0.0013 & \\
\hline & $121-130$ & -0.0044 & -0.0722 & 0.0500 & \\
\hline & $131-140$ & 0.0119 & 0.1250 & 0.1568 & \\
\hline \multirow[t]{10}{*}{1117} & $1-10$ & 0.1362 & 4.6346 & \begin{tabular}{|l|}
0.8733 \\
\end{tabular} & yes \\
\hline & $11-20$ & $4 \mathrm{E}-5$ & 2.5483 & $8 \mathrm{E}-6$ & \\
\hline & $21-30$ & -0.0059 & 2.6432 & 0.2085 & \\
\hline & $31-40$ & $\overline{0.0014}$ & 2.5317 & 0.0139 & \\
\hline & $41-50$ & 0.0053 & 2.0108 & 0.2702 & \\
\hline & $51-60$ & -0.0075 & 1.6822 & 0.3514 & \\
\hline & $61-70$ & 0.0010 & 1.8067 & 0.0114 & \\
\hline & $71-80$ & 0.0069 & 5.1939 & 0.0815 & \\
\hline & $81-90$ & 0.0044 & 4.6306 & 0.0824 & \\
\hline & $76-85$ & 0.0066 & 6.2686 & 0.1421 & \\
\hline \multirow[t]{13}{*}{1107} & $1-10$ & 0.1377 & 7.0387 & 0.8552 & yes \\
\hline & $11-20$ & -0.0415 & 8.9355 & 0.6975 & yes \\
\hline & $21-30$ & -0.0141 & 8.9836 & 0.1604 & \\
\hline & $31-40$ & -0.0306 & 9.1673 & 0.6683 & yes \\
\hline & $41-50$ & 0.0169 & 9.1031 & 0.1735 & \\
\hline & $51-60$ & 0.1246 & 10.7618 & 0.9559 & yes \\
\hline & $61-70$ & 0.0088 & 1.4563 & 0.2383 & \\
\hline & $71-80$ & 0.0011 & 2.3654 & 0.0053 & \\
\hline & $81-90$ & -0.0003 & 4.9779 & 0.0002 & \\
\hline & $91-100$ & 0.0099 & 1.1450 & 0.0607 & \\
\hline & $101-110$ & -0.0157 & 7.4953 & 0.3070 & \\
\hline & $111-120$ & -0.0030 & 5.4728 & 0.0312 & \\
\hline & $121-128$ & 0.0032 & 5.4921 & 0.0328 & \\
\hline
\end{tabular}




\section{Conclusions}

Moisture profiles from neutron probe data provide valuable data in site characterization and to supplement ground water monitoring efforts. Estimates of recharge and its spatial and temporal variability can be made, when the data is used in conjunction with additional data, such as chloride and isotope profiles [Newman, 1996] or hydrogeologic transport properties of the matrix [Vold, 1996]. The neutron probe precision error (reproducibility) is found to be about $0.2 \mathrm{vol} \%$ under in-situ field conditions where the slope in moisture content with depth is varying slowly. This error is about 2 or 3 times larger near moisture spikes (near the vapor phase notch), due to the sensitivity of the probe response to vertical position errors on the order of $0.5^{\prime \prime}$.

Calibrations were performed to correct the downhole probe response to the volumetric moisture content determined on core samples. Calibration is sensitive to borehole diameter and casing type, requiring 3 separate calibration relations for the boreholes surveyed here. Power law and linear fits give similar calibration results except in the driest regions where linear fits can give negative moisture content, so power law fits were used for the results reported in this study.

Findings in the boreholes reported here confirm the broad features seen previously in moisture profiles at Area G, a wetter near-surface region with large variability, a very dry region at greater depths, and a moisture spike at the vapor phase notch (VPN), near the interface between the vitrified and devitrified stratigraphic units near the base of the mesa. Comparing borehole profiles from several locations, moisture content is qualitatively observed to decrease as one approaches the mesa edge, showing a trend consistent with mesa edge drying of the interior.

Borehole moisture shows significant seasonal variations in time at near-surface locations with maximum contents in the fall following the summer showers and in the spring corresponding with the time of snow melt. These distinct variations (several percent volumetric content) are restricted to the uppermost depths in the borehole profiles, 6-10' only. Below this depth, the moisture profiles are approximately constant in time, however, some variations at these depths can be discerned as statistically significant by appropriately averaging over spatial intervals and considering trends over long times ( 1 year). These variations are small enough that the influence on the accuracy of the moisture calibration procedure is negligible. However, with a fixed calibration, the moisture vatiations in time are large enough that trends over depth intervals and over time are found to be statistically significant over the obsevation period in this study from the summer of 1996 to the summer of 1997.

Specifically, moisture profiles appear to be impacted by disposal operations at least at one location (1121) and probably at a second borehole (1107). At 1121, the moisture increases seen at all depth intervals down to 70' correspond to an average increase of hydraulic conductivity (estimate for the 
recharge rate under unit gradient conditions) of several $\mathrm{mm} / \mathrm{yr}$. At 1107 , a moisture increase corresponding to a recharge estimate of order $1-2 \mathrm{~cm} / \mathrm{yr}$ is significant at the 50-60' depth interval. In pit 37, a moisture pulse associated with a 'bath-tubbing' effect due to grading operations near the moisture monitor port was observed which is large compared to the nominal recharge rates expected at Area G. This is discussed in detail in a separate report. [Vold, 1997]. Moisture profiles at the drier conditions near the mesa edge (1117) do not appear to be significantly influenced by disposal operations.

Continued and improved moisture monitoring programs are recommended to assure the actual site performance conforms to the assumptions made in the Performance Assessment analyses in the wake of continuing impact from ongoing disposal operations.

\section{Acknowledgments}

This work was supported in part by the U.S Department of Energy, Office of Waste Management. The author thanks Mike Borduin who collected most of the neutron probe field data, and also Mark Haggenstad, Dennis Newell and David Loaiza who contributed to the collection of the field data.

\section{References}

[Bevington,1969] Bevington, P.R., Data Reduction and Error Analysis for the Physical Sciences, McGraw Hill Book Co., New York, 1969.

[DBSA,1995] - D. B. Stephens and Associates, "Laboratory analysis of soil hydraulic properties of G-5 soil samples, report no. DBSA/3854/195-1", prepared for Los Alamos National Laboratory, Los Alamos, New Mexico, January 1995.

[Hollis, et.al., 1997] Hollis, Diana, Vold, Erik L., Shuman, Rob, Birdsell, Kay H., Bower, Kathleen, Newman, B. D., Springer, Everett P., Hansen, Wayne, Longmire, P. A., Rogers, David B., Krier, D. J., 'Performance Assessment And Composite Analysis For Los Alamos National Laboratory Material Disposal Area G', Los Alamos National Laboratory Report LA-UR-97-0085, Los Alamos, NM, USDOE LANL Area G Performance Assessment, Jan. 1997.

[Krier, et.al.,95] D. Krier, P. Longmire, R. Gilkeson, H.J.Turin, "Geologic, Geohydrologic, and Geochemical Data Summary of MDA G, TA-54, LANL", Los Alamos National Laboratory Report LA-UR-95-2696, (see also App.2A to Ref.1), Los Alamos, NM, 1995.

[Krier, Marin, Kisiel, 1996] personal communication, to be published in LANL ER Project documents. 
[Loaiza and Vold 1995] Loaiza, D. and Vold, E., Moisture Profile Measurements in Subsurface Monitor Holes at the Los Alamos LLRW Disposal Site, Los Alamos National Laboratory Report LAUR-95-1922, Los Alamos, NM, June, 1995.

[Newell, 1997] Dennis Newell, EM-SWO, LANL, personal communication 1997.

[Newman, 1996] B. D. Newman, "Vadose Zone Water Movement at Area G, LANL, TA-54: Interpretations Based on Chloride and Stable Isotope Profiles", Los Alamos National Laboratory Report LA-UR-96-4682, Los Alamos, NM, 1996.

[Puglisi and Vold,1995] Puglisi, C.V. and Vold, E.L., Low Impact Sampling under a LLW Disposal Pit using Horizontal Drilling Technology, Los Alamos National Laboratory Report LAUR-95-2060, Los Alamos, NM, proceedings of the 1995 Materials Research Society Fall Meeting, Symposium on the Scientific Basis for Nuclear Waste Management XIX, Boston, Nov.1995.

[Stephens,1996] Daniel B. Stephens, Vadose Zone Hydrology, CRC Press, Baca Raton, FL, 1996.

[Vold,1996] Vold, E.L., Analysis of Liquid Phase Transport in the Unsaturated Zone at a Mesa Top Disposal Facility, Los Alamos National Laboratory Report LA-UR-96-370, Los Alamos, NM, 1996.

[Vold, 1997] E. Vold, Synopsis of Some Preliminary Computational Studies Related to Unsaturated Zone Transport at Area G, report in progress as a Los Alamos National Laboratory Unclassified Report, Dec. 1997.

[Walpole and Myers, 1978] Walpole and Myers, 2nd Ed, p.289, Probability and Statistics for Engineers and Scientists, MacMillan, New York, NY, 1978.

[Wilson, Everett, Cullen, 1995] L.G. Wilson, L.G. Everett, S.J. Cullen, Vadose Zone Characterization and Monitoring, Lewis Publishers, Ann Arbor, MI, 1995. 


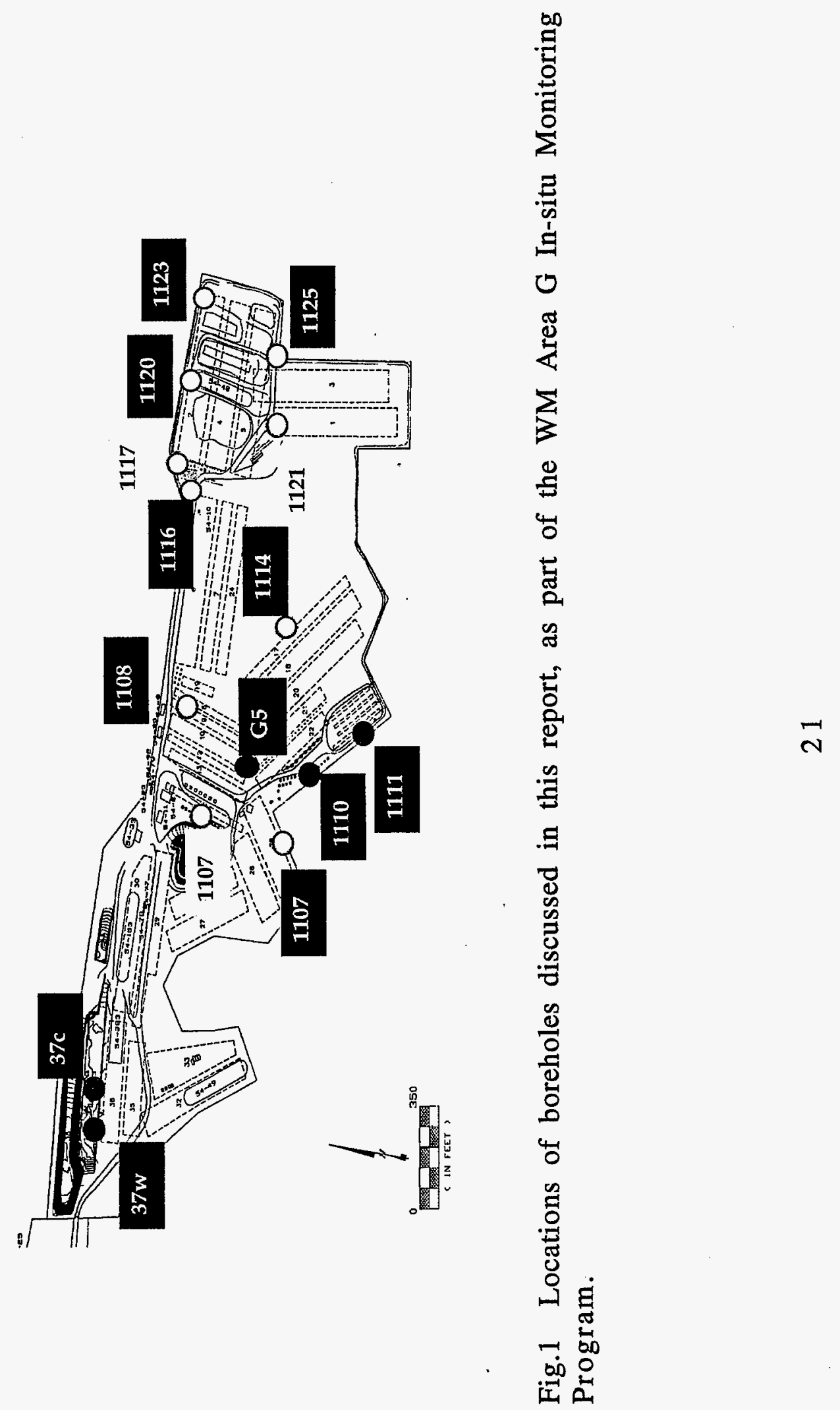



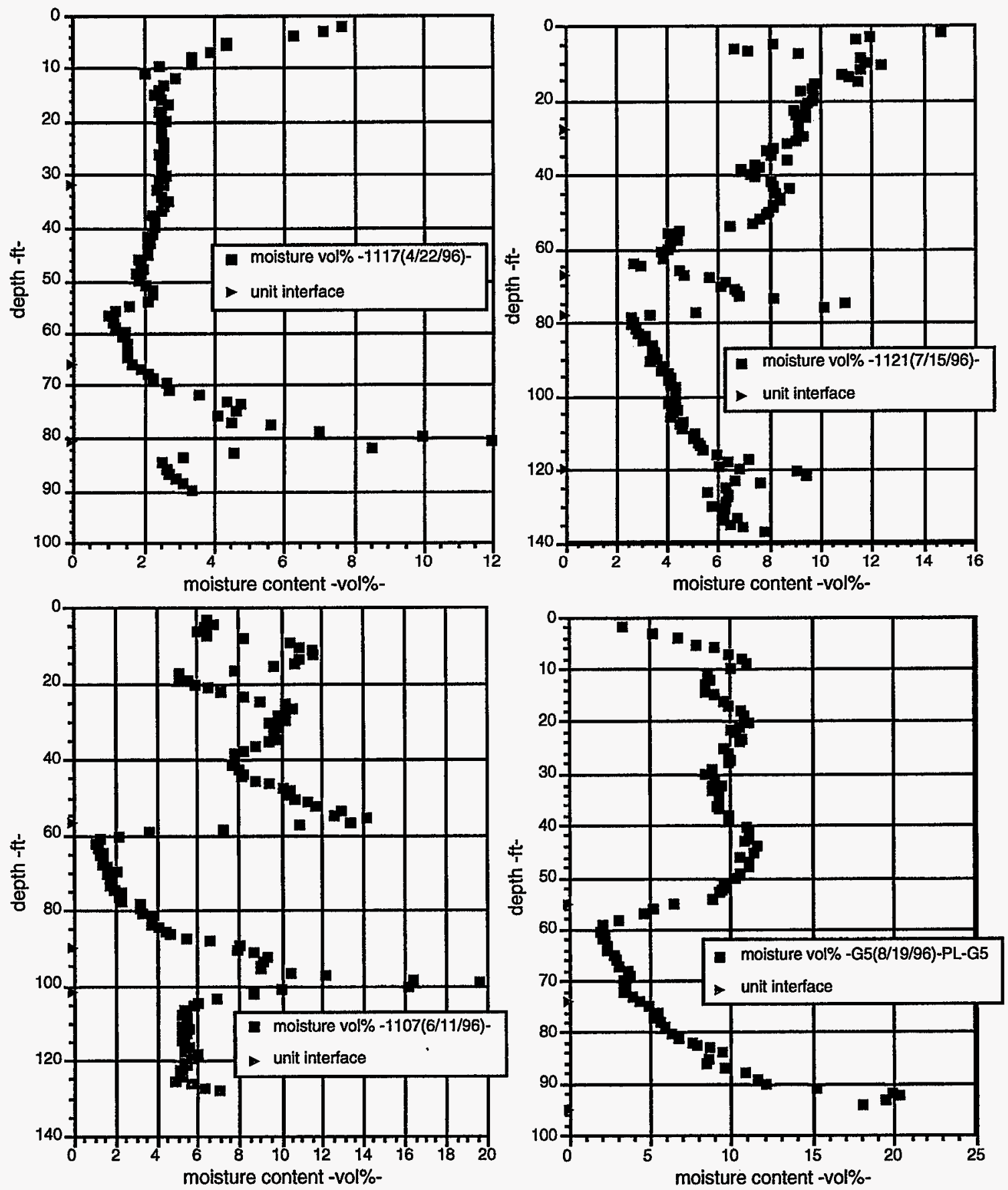

Fig.2 Calibrated moisture profiles (vol\%) measured in the four boreholes used in the in-field calibration procedure described in the text. 


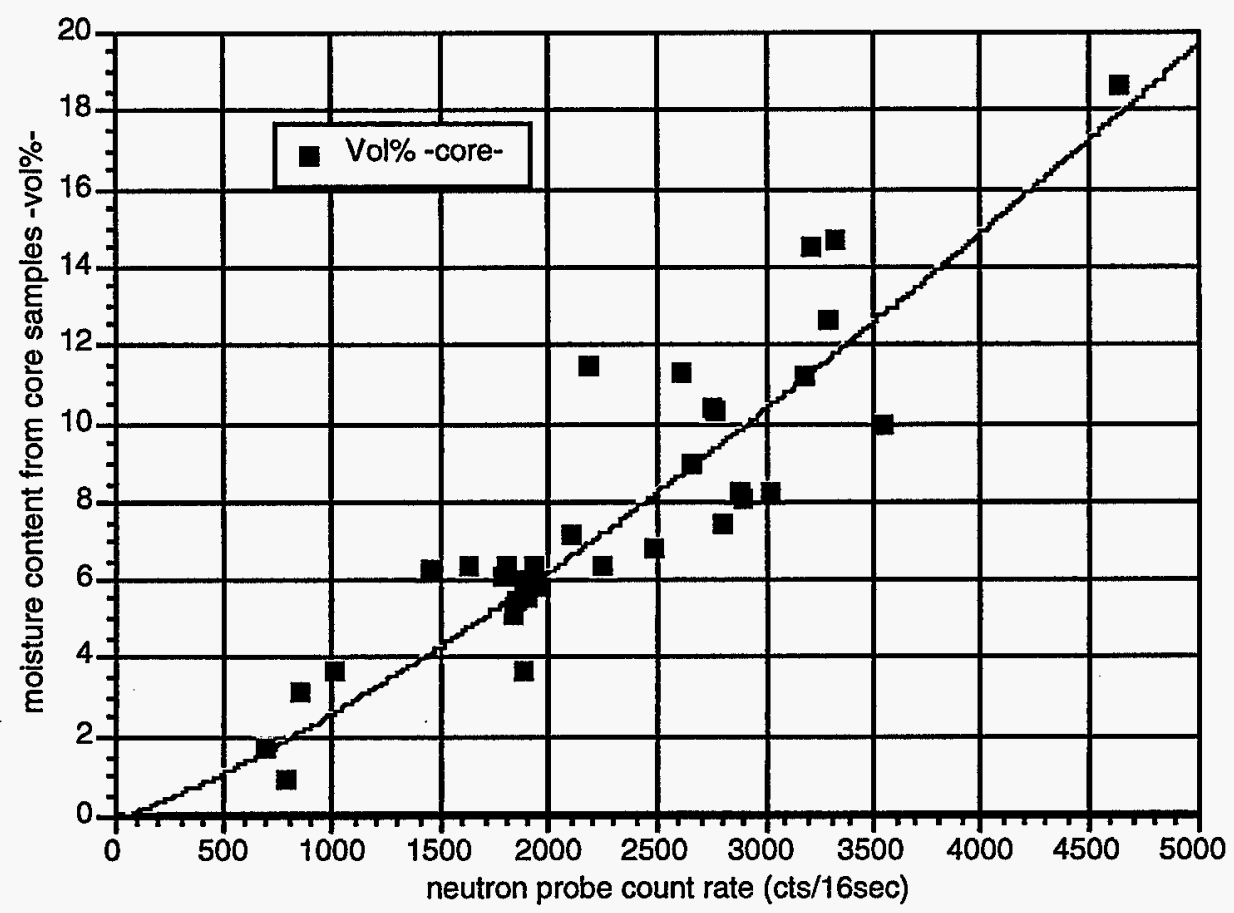

Fig. 3 Moisture content calibration curve for borehole 1107 alone.

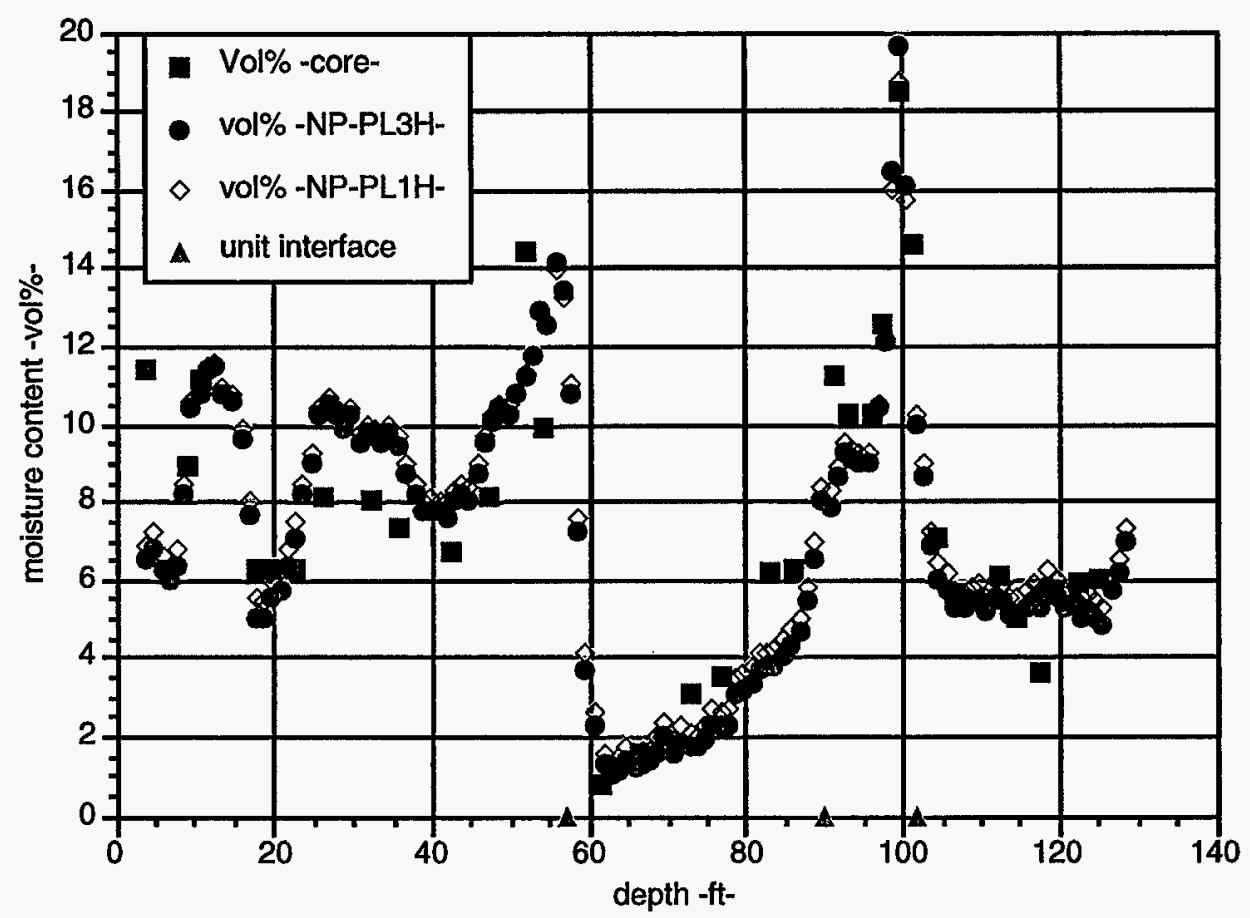

Fig. 4 Moisture content profile for borehole 1107 comparing results from the core samples, the power law calibration curve to the 1107 data alone (PL1H) and the power law calibration curve for the 36 " holes (PL3H). 


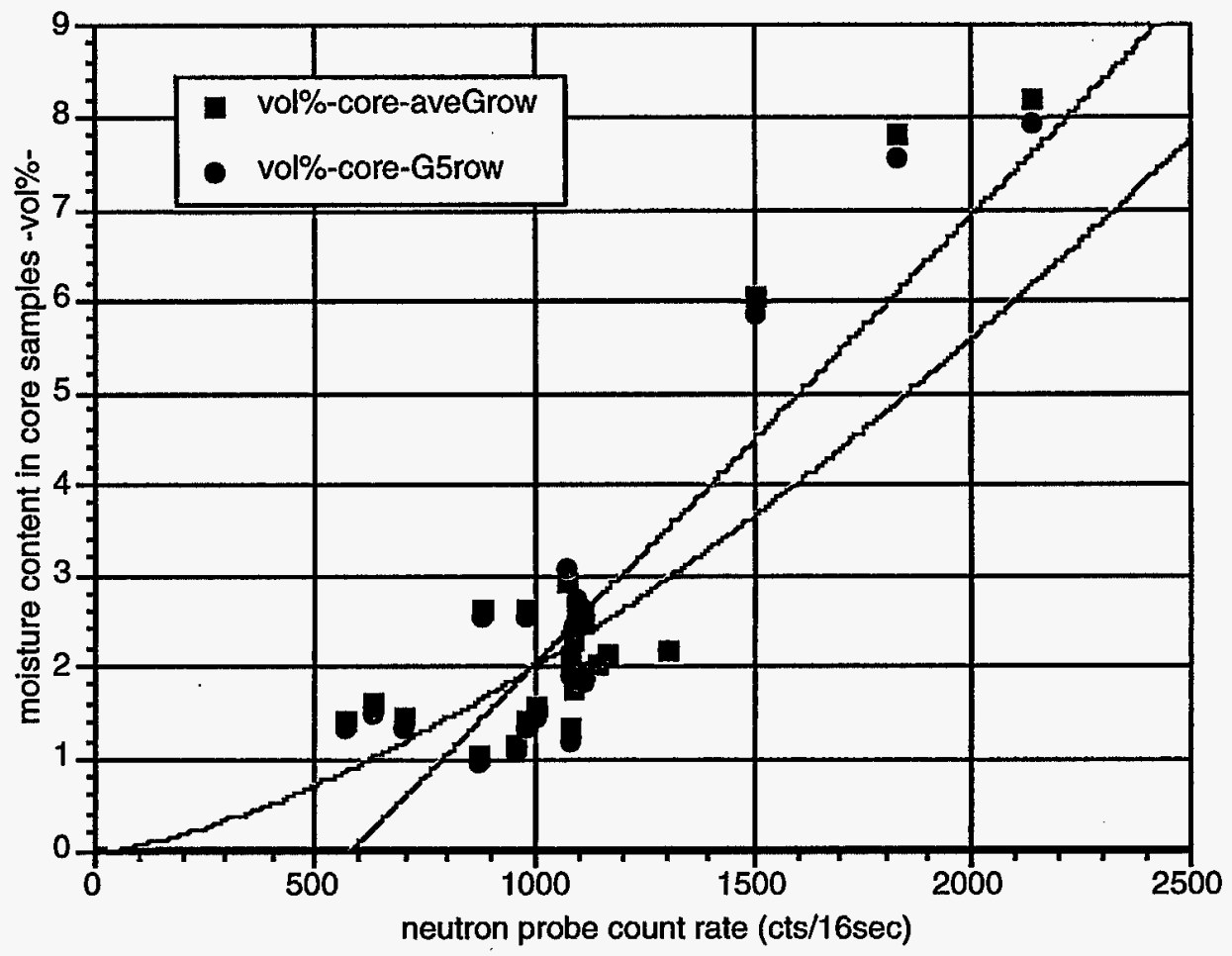

Fig. 5 Moisture content calibration curve for borehole 1117 alone, with moisture content in core samples based on Area $G$ average stratigraphic bulk densities (aveGrow) and based on borehole G-5 bulk density values.

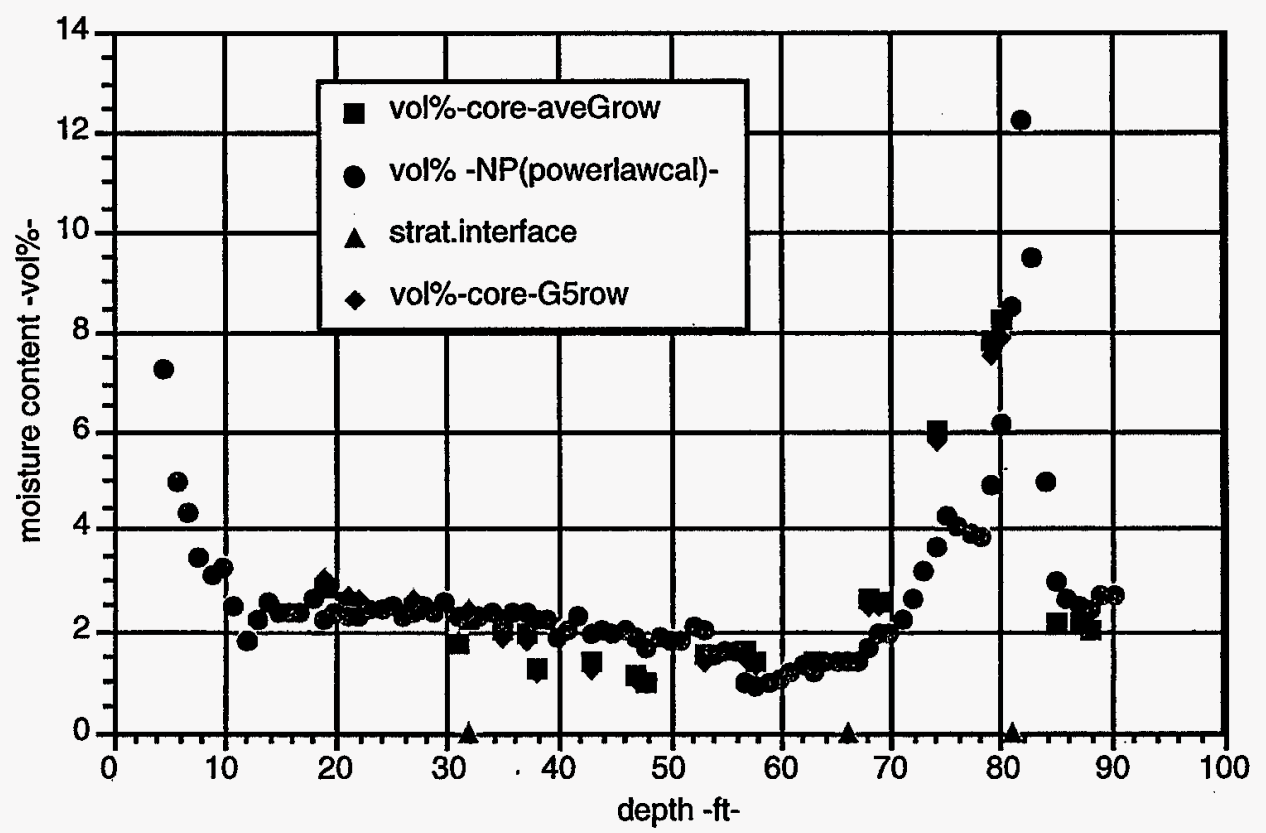

Fig. 6 Moisture content profile for borehole 1117 comparing results from the core samples volumetric content using two estimates of the bulk density (see Fig.5) and to the power law calibration curve to the 1117 data alone. 


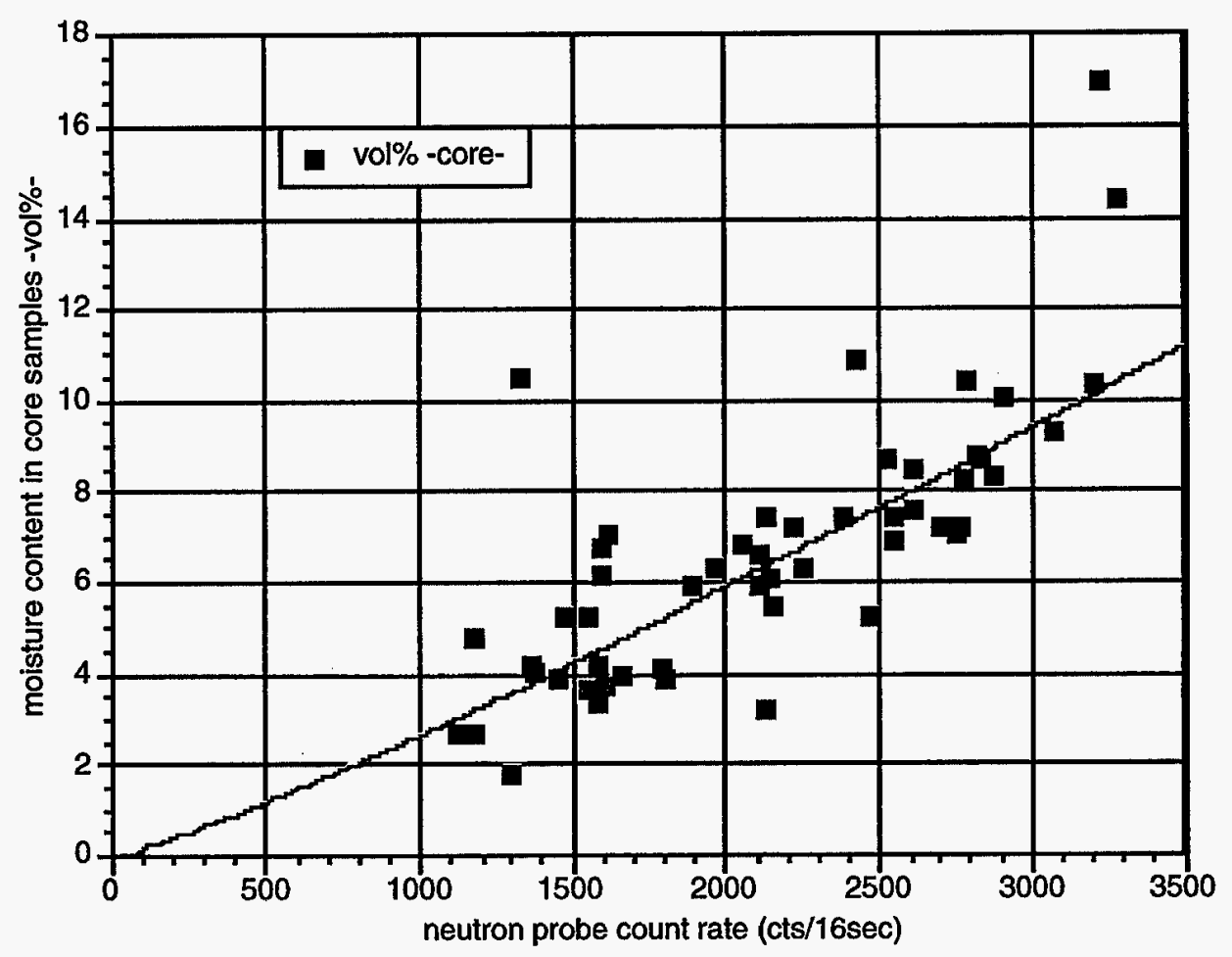

Fig. 7 Moisture content calibration curve for borehole 1121 alone, showing the power law fit to the data.

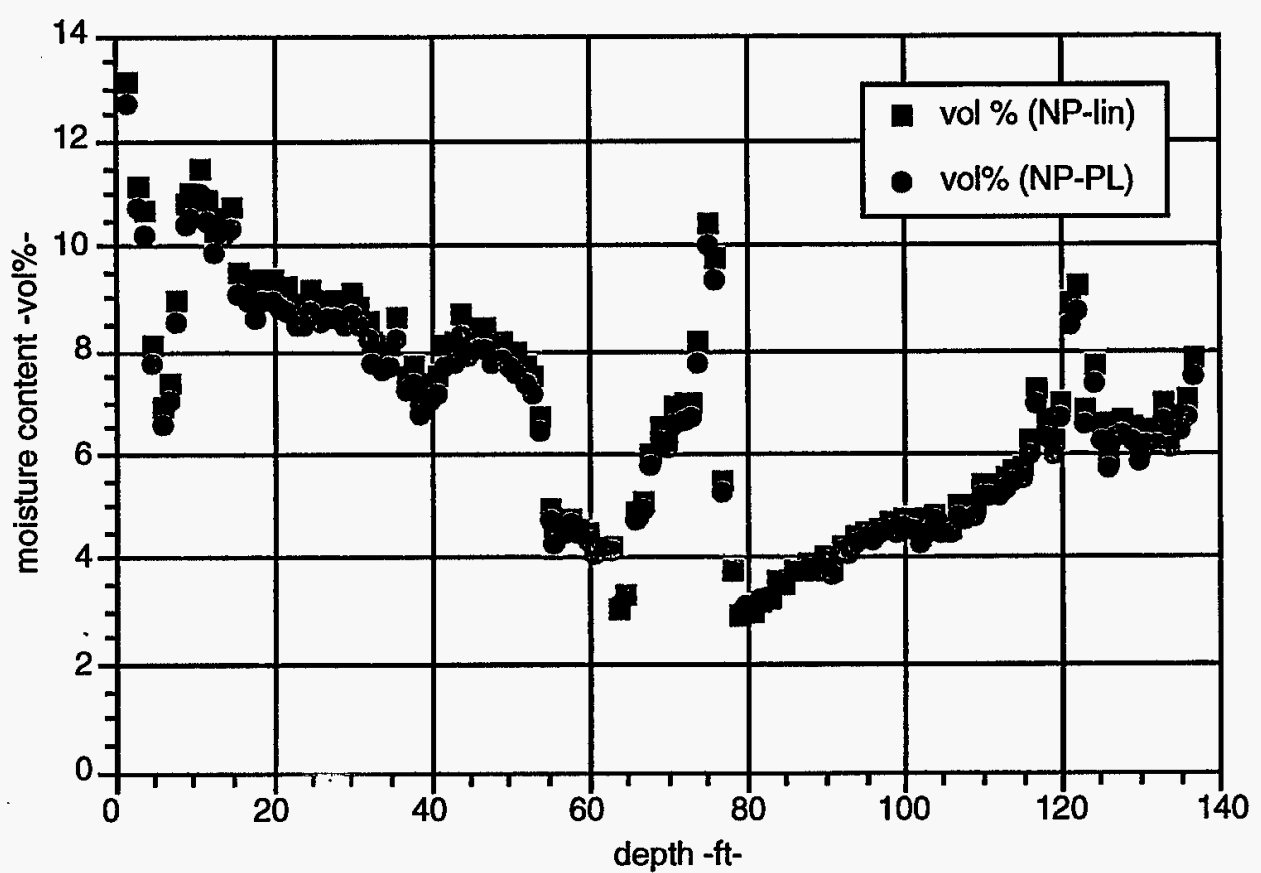

Fig. 8 Moisture content profile for borehole 1121 comparing neutron probe results from the power law calibration curve (NP-PL) to the 1121 data alone with a linear fit to the 1121 borehole data (NP-lin). 


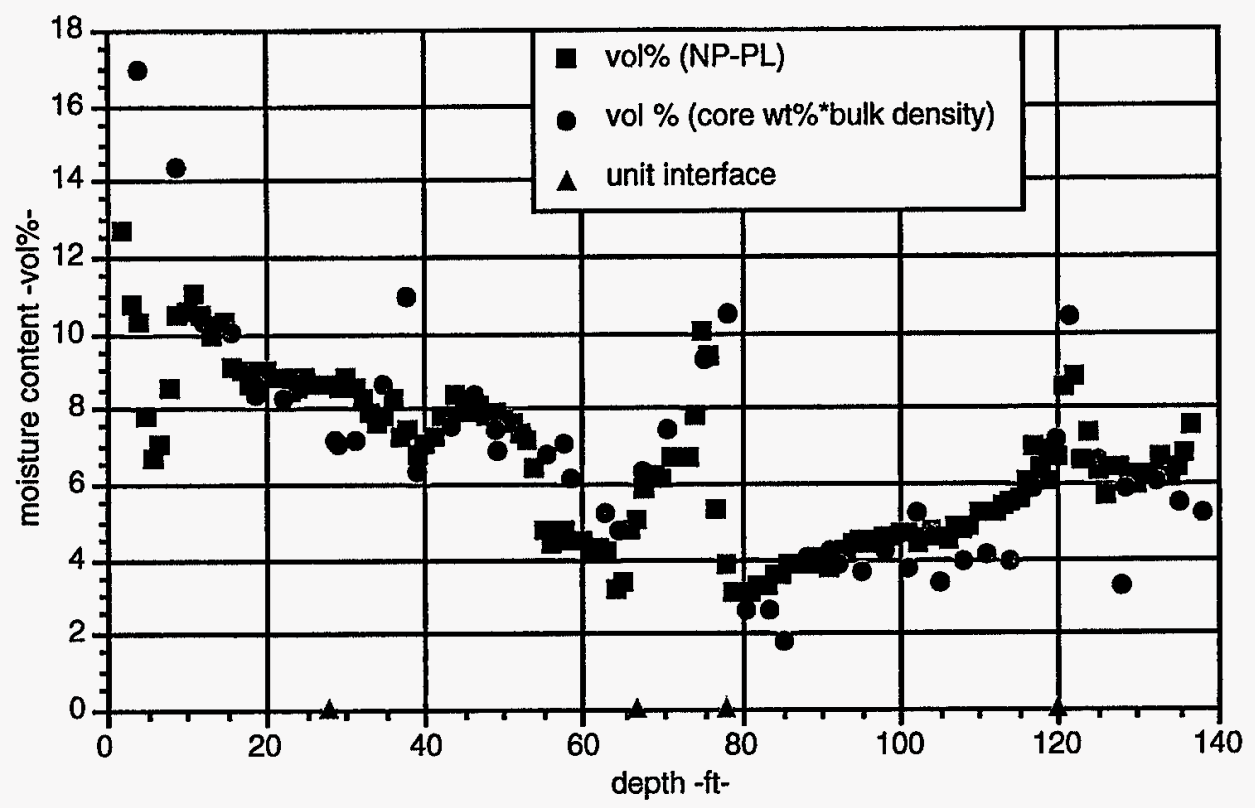

Fig. 9 Moisture content profile for borehole 1121 comparing results from the core samples volumetric content (core ${ }^{w t} \%$ *bulk density) to the power law calibration curve to the 1121 data alone.

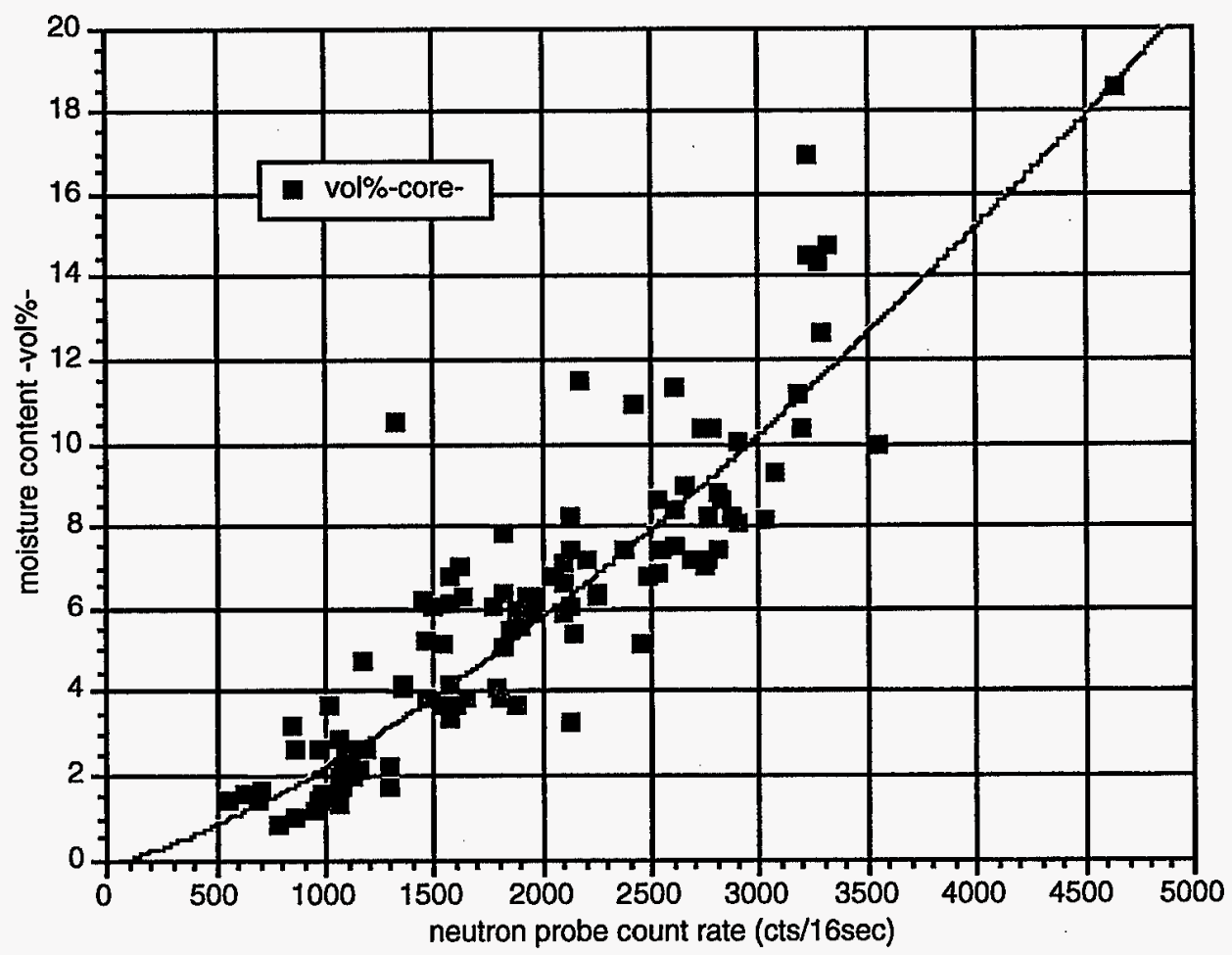

Fig. 10 Calibration curve for data combined from three boreholes (54-1107,$1117,-1121$ ) showing corrected moisture content (vol\%-core derived from core sample weight\% data) verses in-situ neutron probe count rate (cts/16sec standard count interval) taken at the same locations as each core sample. 


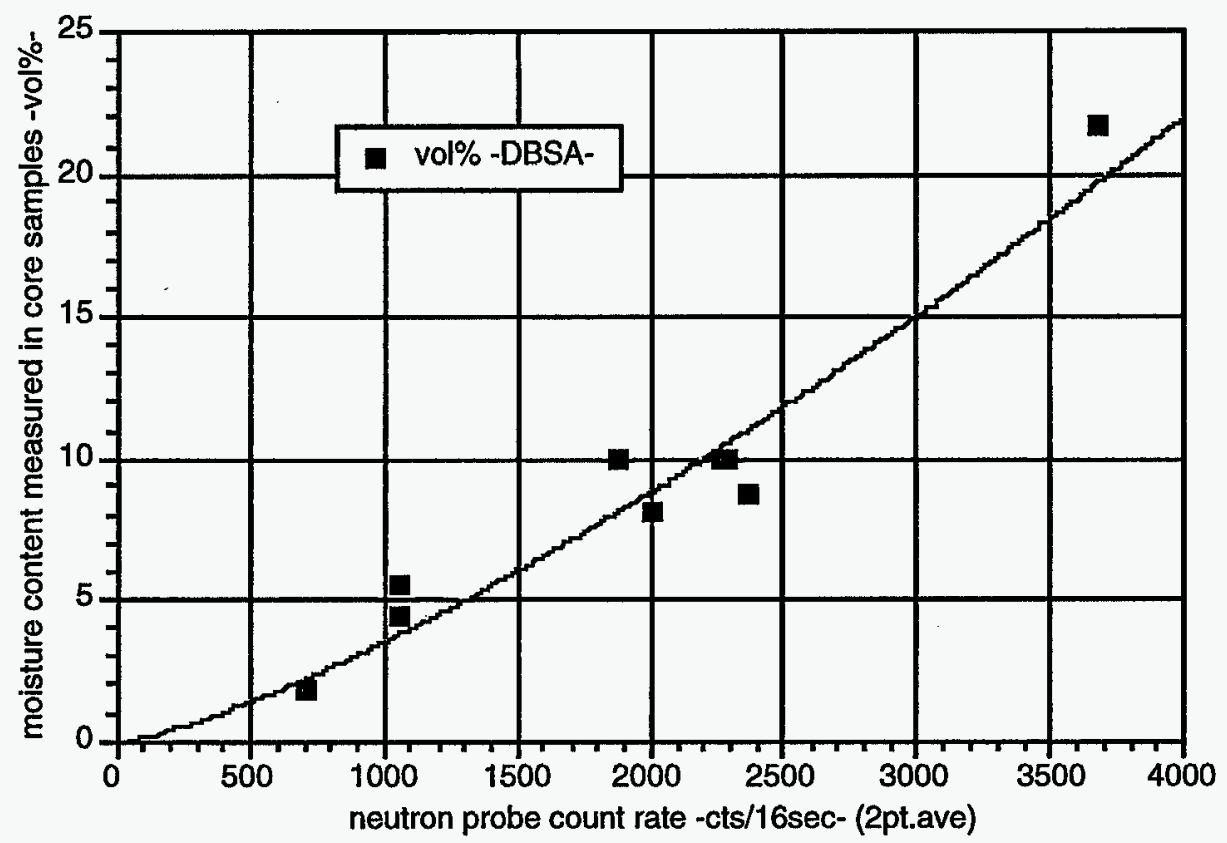

Fig. 11 Calibration curve for data from borehole G-5 showing moisture content measured on core samples (vol\%-DBSA) verses in-situ neutron probe count rate (cts/16sec standard count interval) taken at the same locations as each core sample. Calibration applies to boreholes G-5, 1110, and 1111.

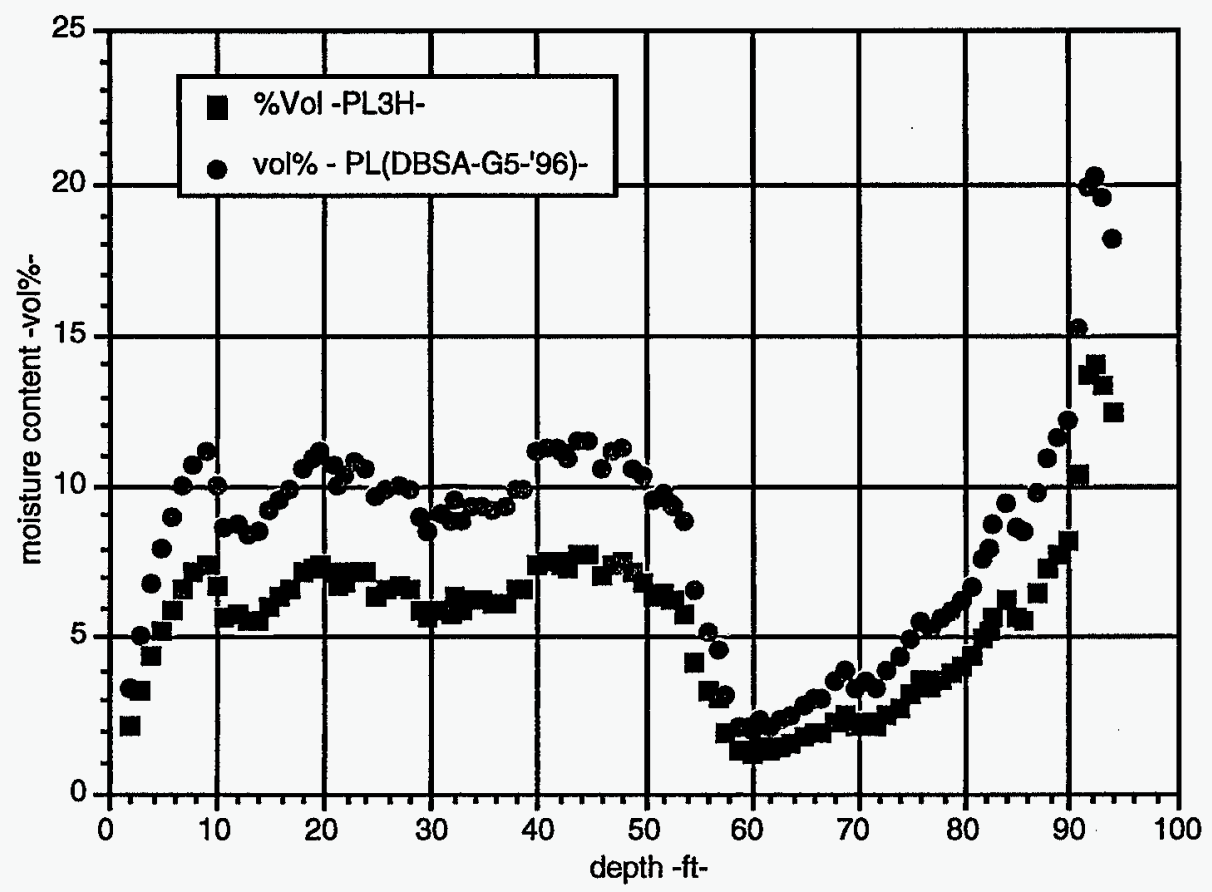

Fig. 12 Moisture content profile for borehole G-5 (4.5" diameter borehole) comparing results from the power law calibration curve from the G-5 data (DBSA-G\%-'96) to the calibration result from the 3 hole, 6" diameter, calibration (PL-3H). 


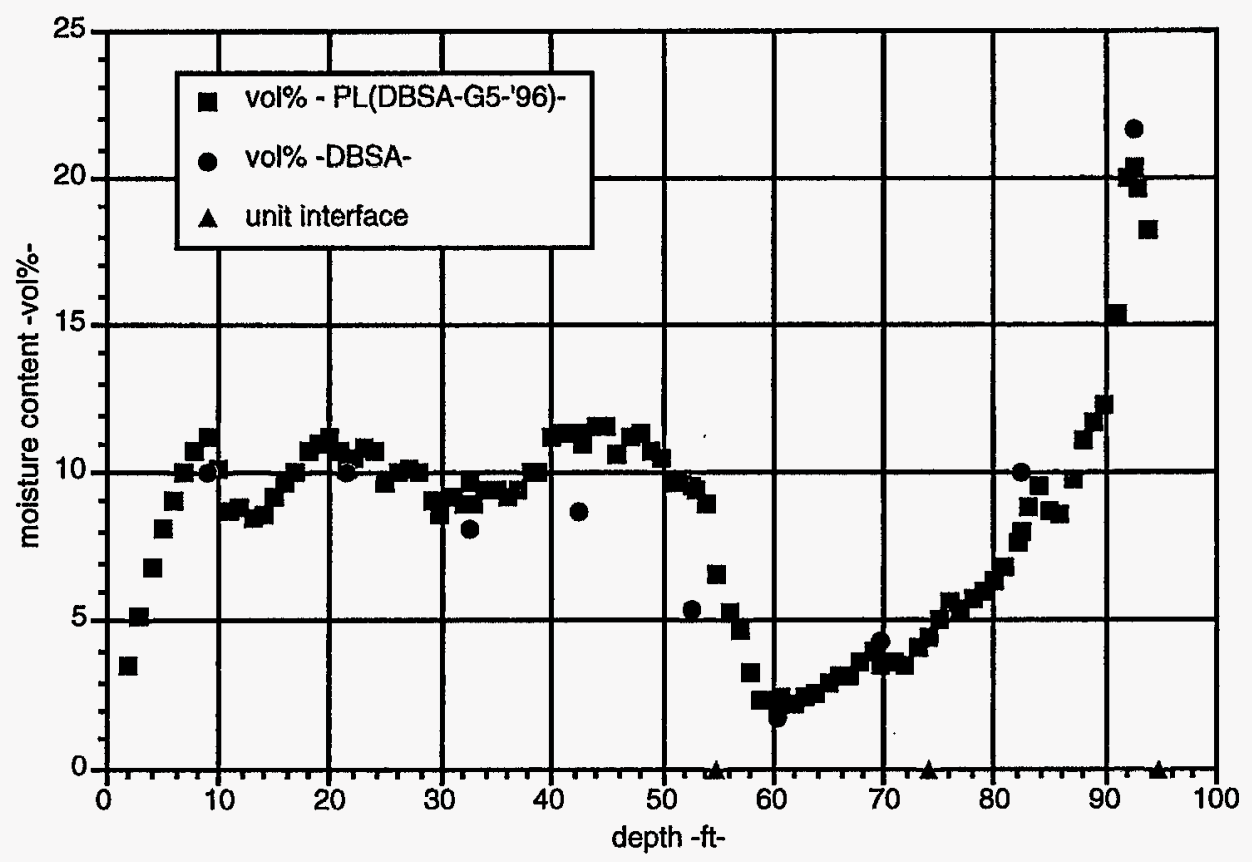

Fig. 13 Moisture content profile for borehole G-5 comparing results from the power law calibration curve from the G-5 data (DBSA-G5-'96) to the core sample results measured after the 1994 drilling (DBSA).

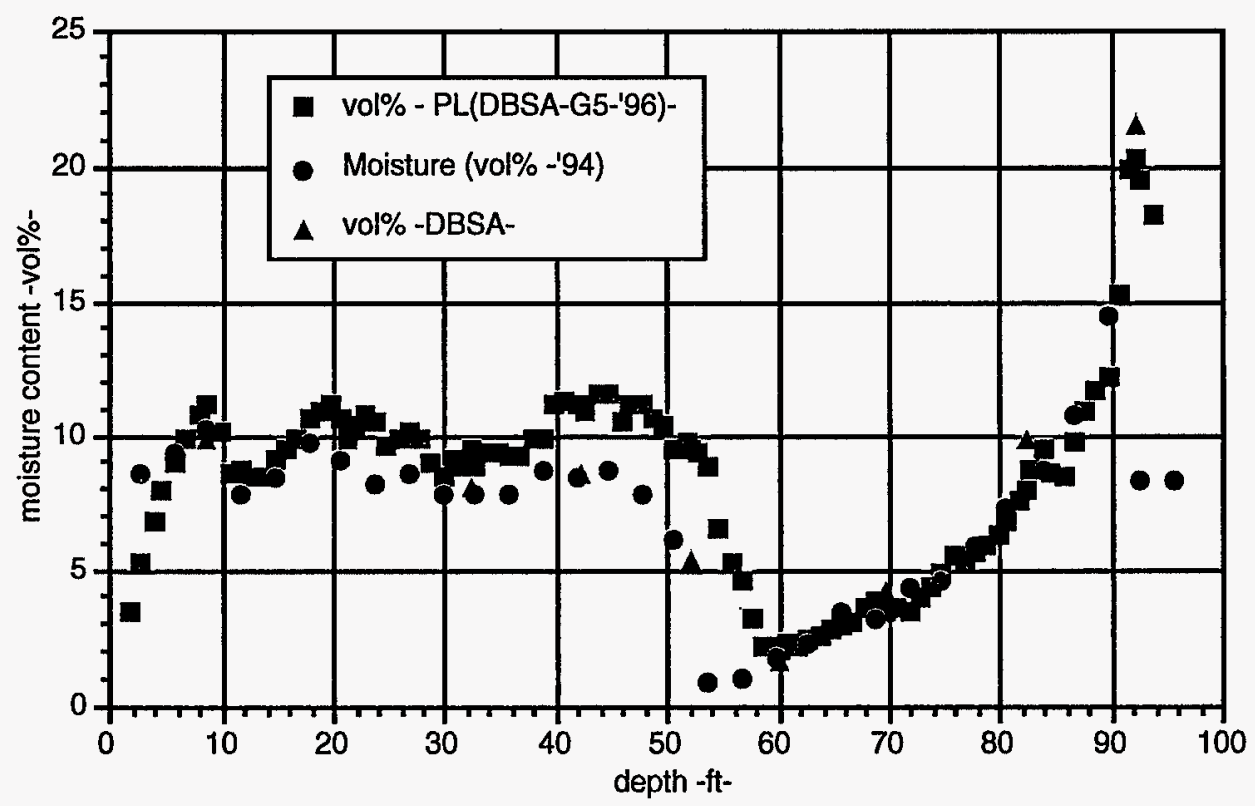

Fig. 14 G5 Moisture content profile for borehole G-5 comparing results from the core samples volumetric content (DBSA) to the power law calibration obtained in 1996 (DBSA-G5-'96) and to that obtained in 1994 (vol\%-'94). 


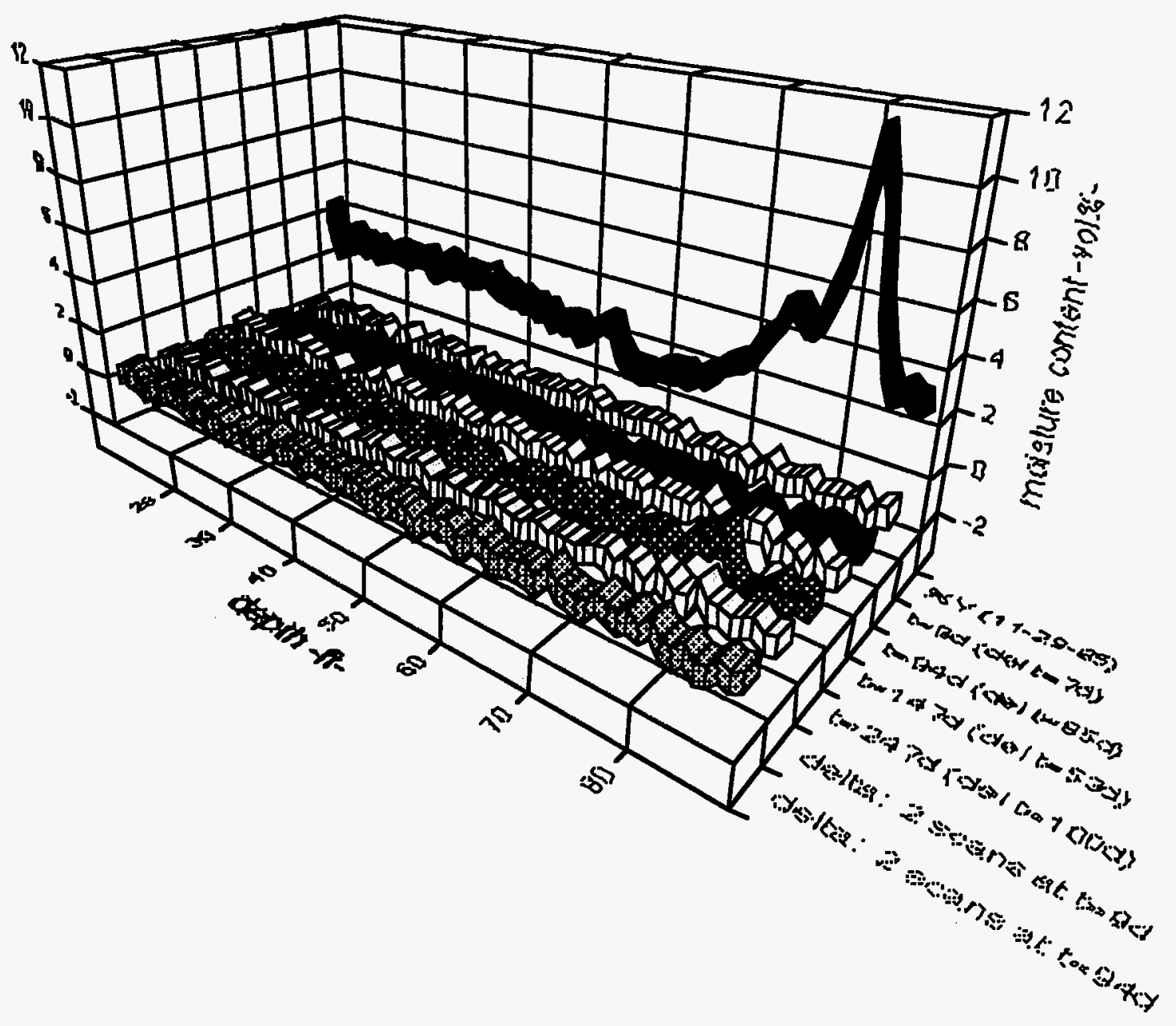

Fig. 15 Initial moisture profile (vol\% on $11 / 29 / 95$ ) in borehole 1117 is shown in the farthest strip. The closer strips show moisture difference between profile measurements made at successive times of $9,94,147$ and 247 days postdrilling. The closest two strips indicate differences between two profile measurements made on the same day $(t=9 d$ and $t=94 d)$, and indicate the magnitude of error associated with in-field reproducibility. 


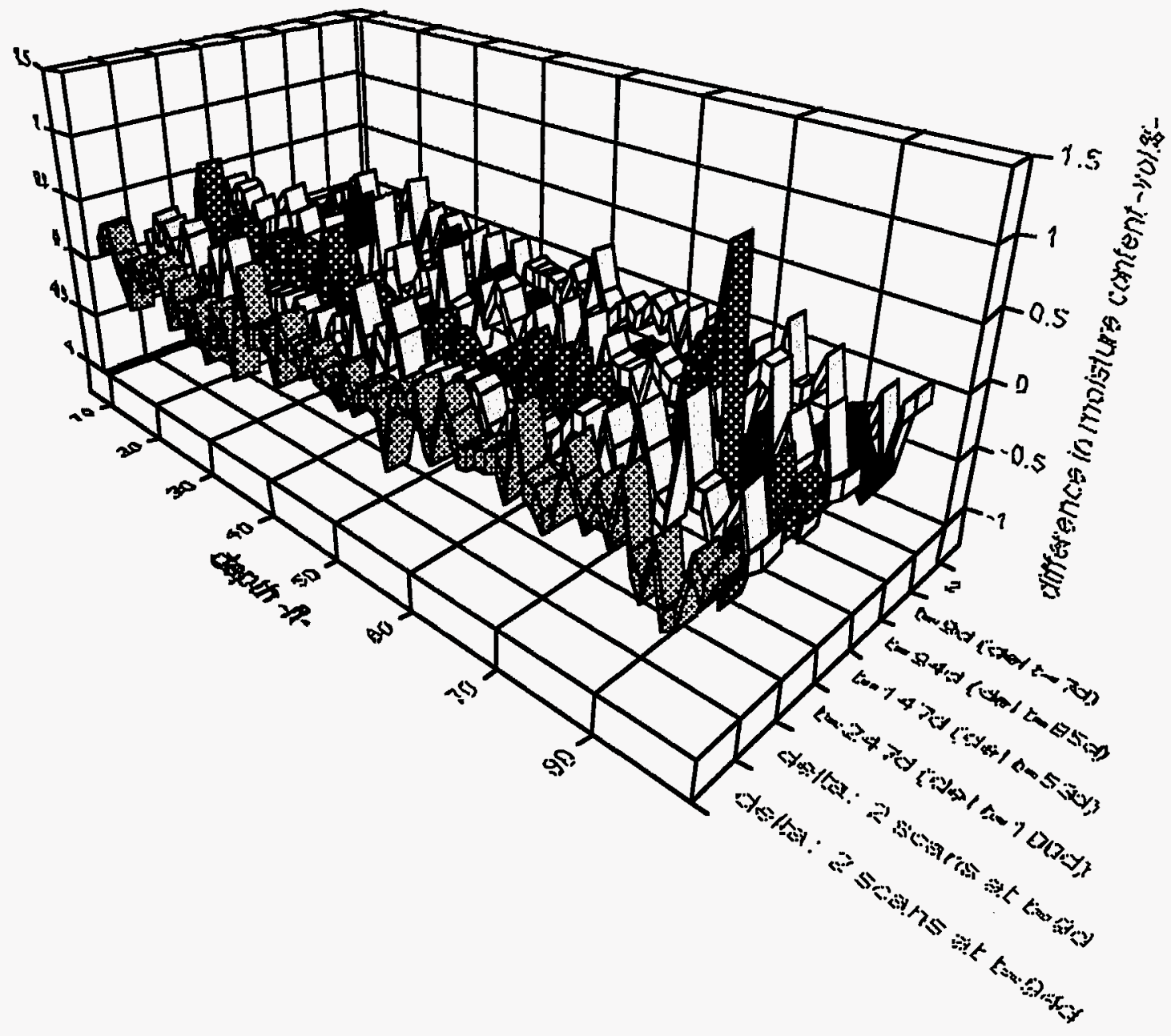

Fig. 16 The moisture difference in borehole 1117 between profile measurements made at successive times of 9, 94, 147 and 247 days, postdrilling. The closest two strips show differences between two profile measurements made on the same day (at $t=9 d$ and $t=94 d$ ), and these indicate the magnitude of error associated with in-field reproducibility. 


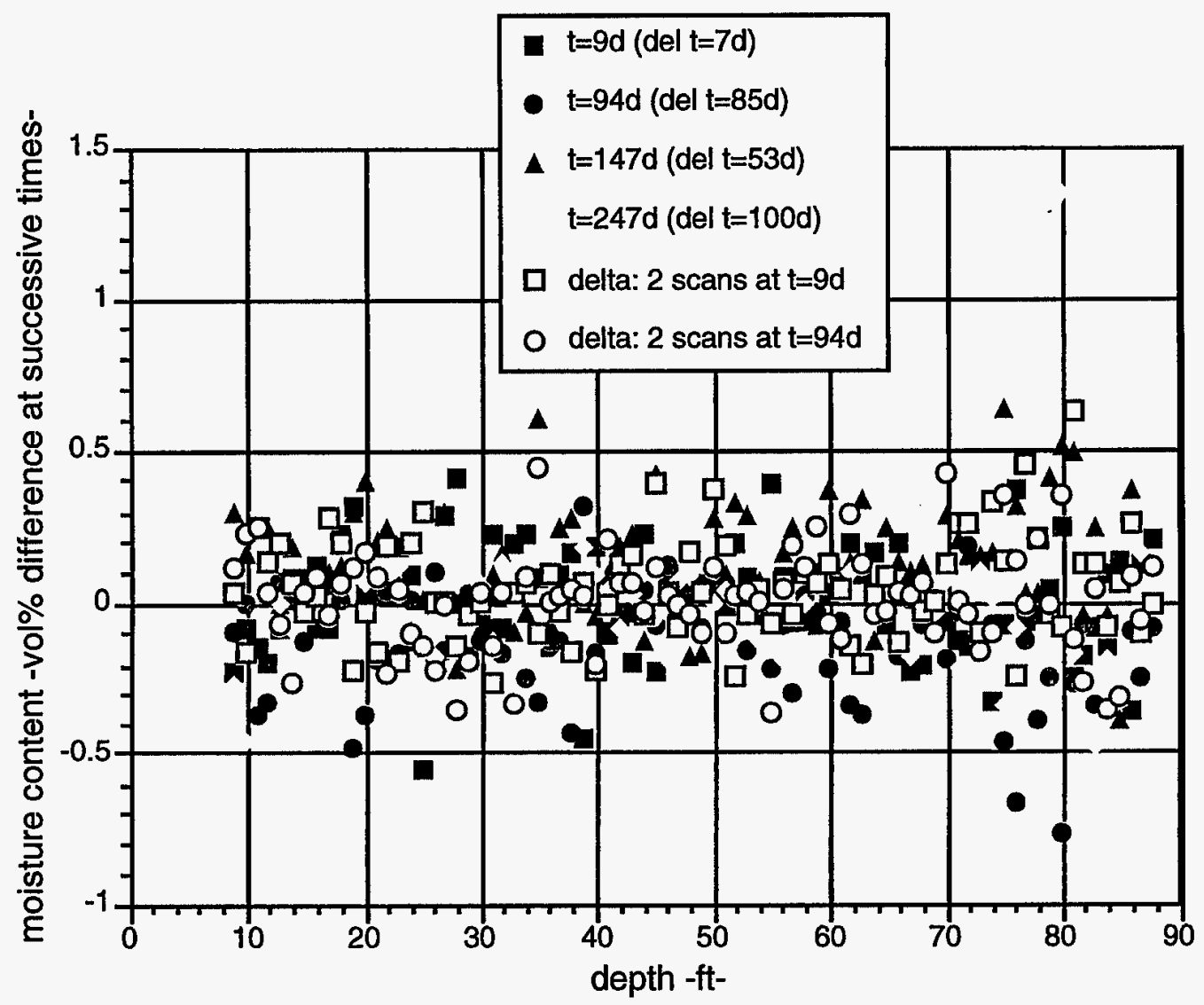

Fig. 17 The moisture difference in borehole 1117 between profile measurements made at successive times (this is the same data as in Fig.16). The symbols labeled 'delta' indicate differences between two profile measurements made on the same day, and indicate the magnitude of error associated with in-field reproducibility. 

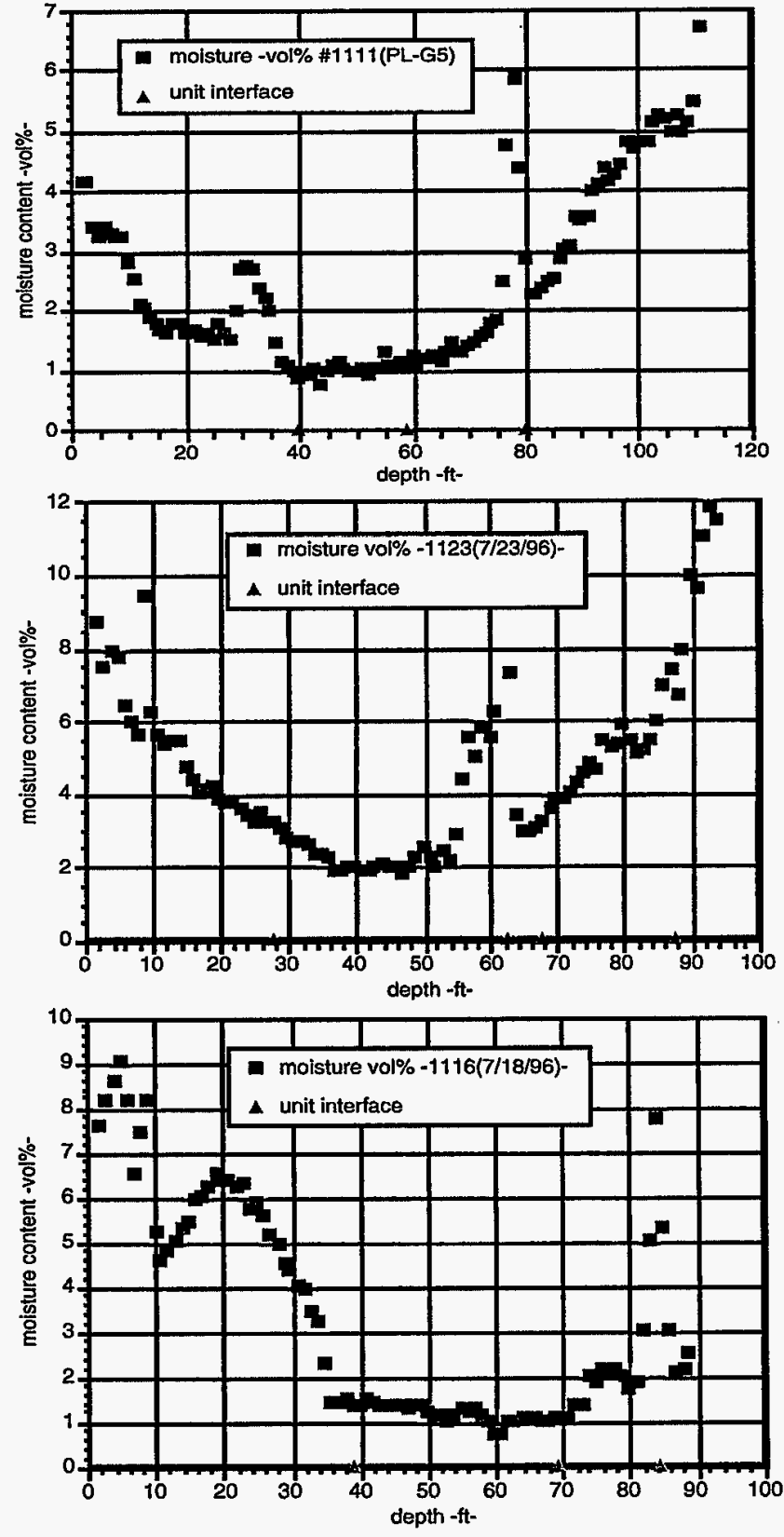

Fig. 18 Moisture profiles for three boreholes in the baseline recharge monitoring program.

Borehole-1111 (4.5" hole calibrated with G-5 data)

Borehole-1123 (4.5" hole calibrated with $3 \mathrm{H}-\mathrm{PL}$ data)

Borehole-1116 (4.5" hole calibrated with $3 \mathrm{H}-\mathrm{PL}$ data)

These boreholes each pass through the VPN (Vapor Phase Notch), as seen by the moisture spike located at depths 78', 62', and 84', respectively. 


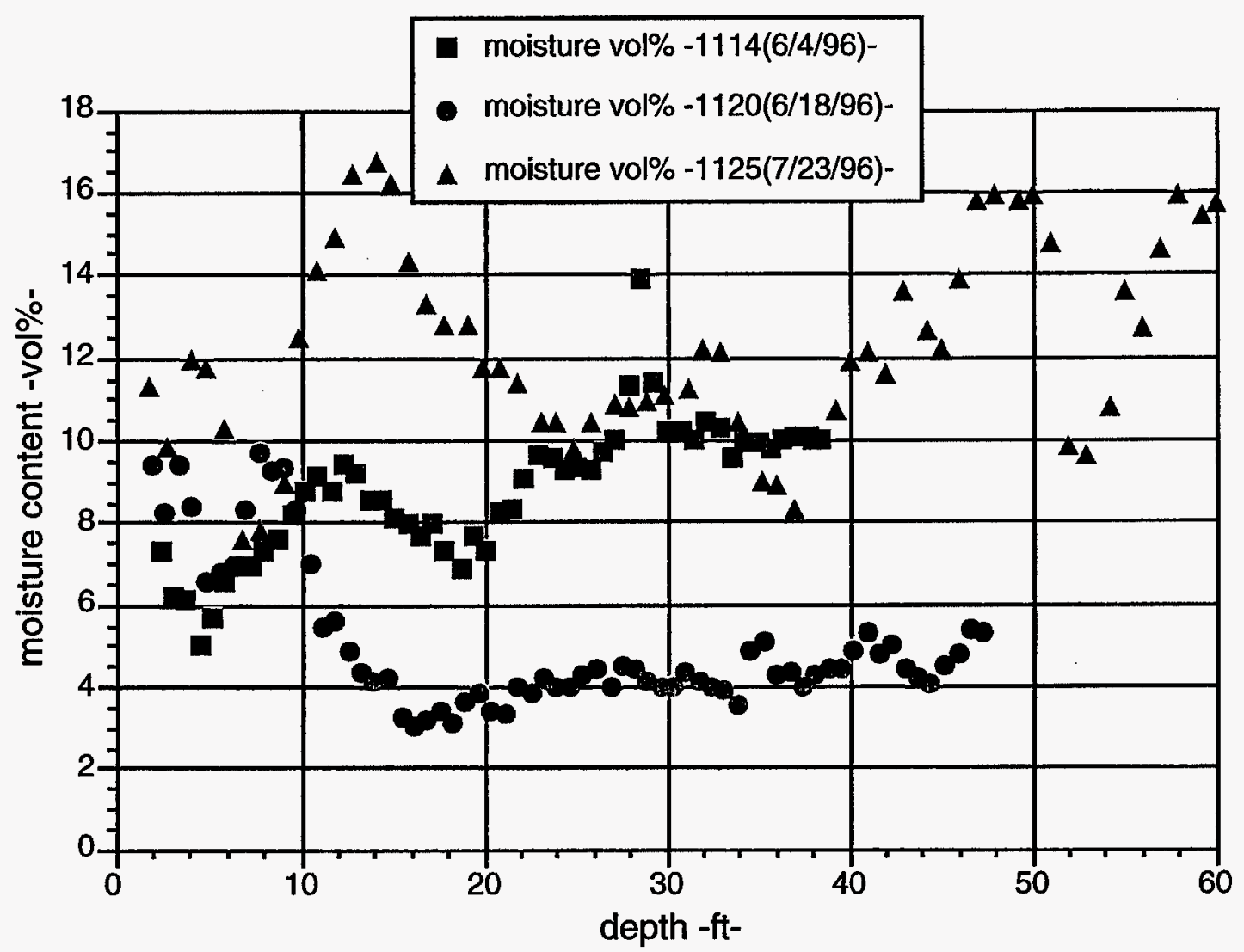

Fig. 19 Moisture profiles for three boreholes in the baseline recharge monitoring program, 1114, 1120, 1125. These are 4.5 " diameter calibrated with the $3 \mathrm{H}-\mathrm{PL}$ fit. 


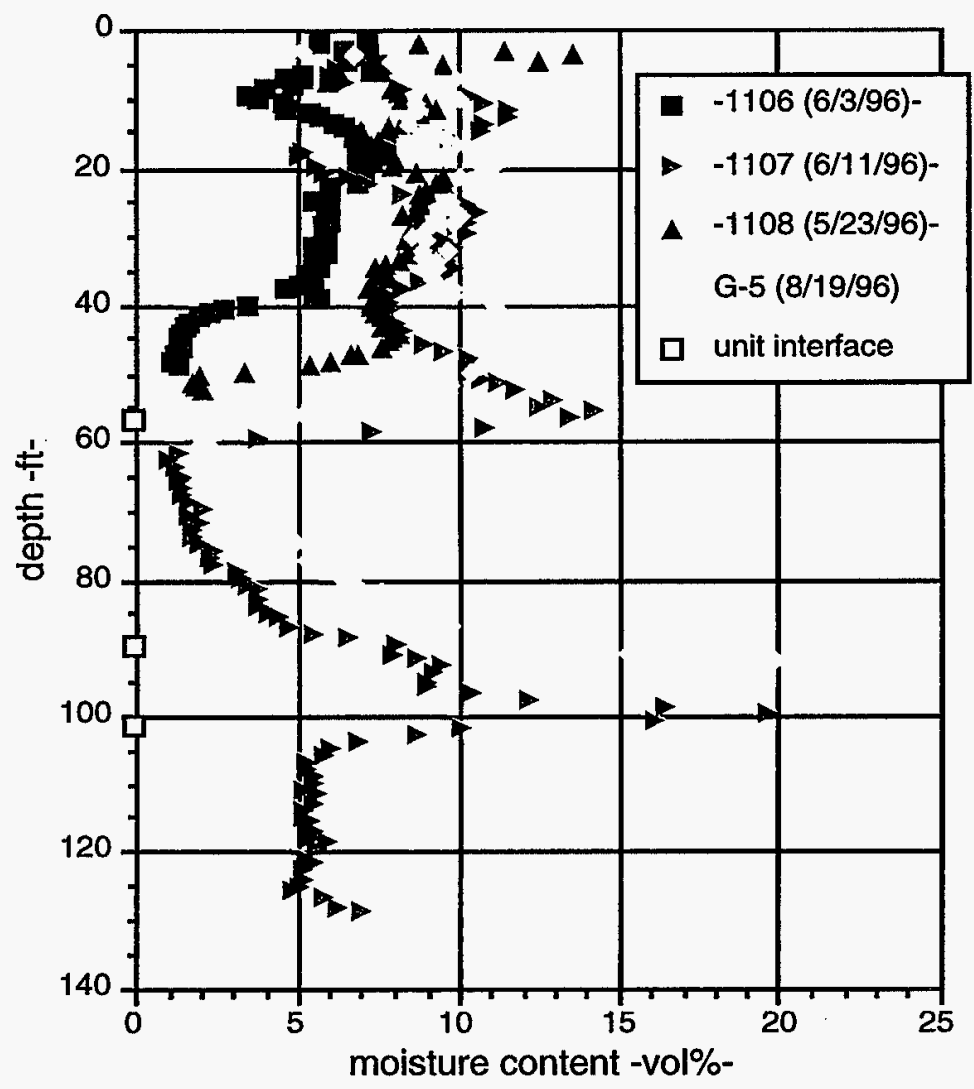

Fig. 20 Moisture profiles for boreholes in the baseline recharge monitoring program, 1106, 1107, 1108, (4.5" diameter calibrated with the 3H-PL fit) and in comparison to G-5. These are centrally located across Area G as seen in Fig. 1. 

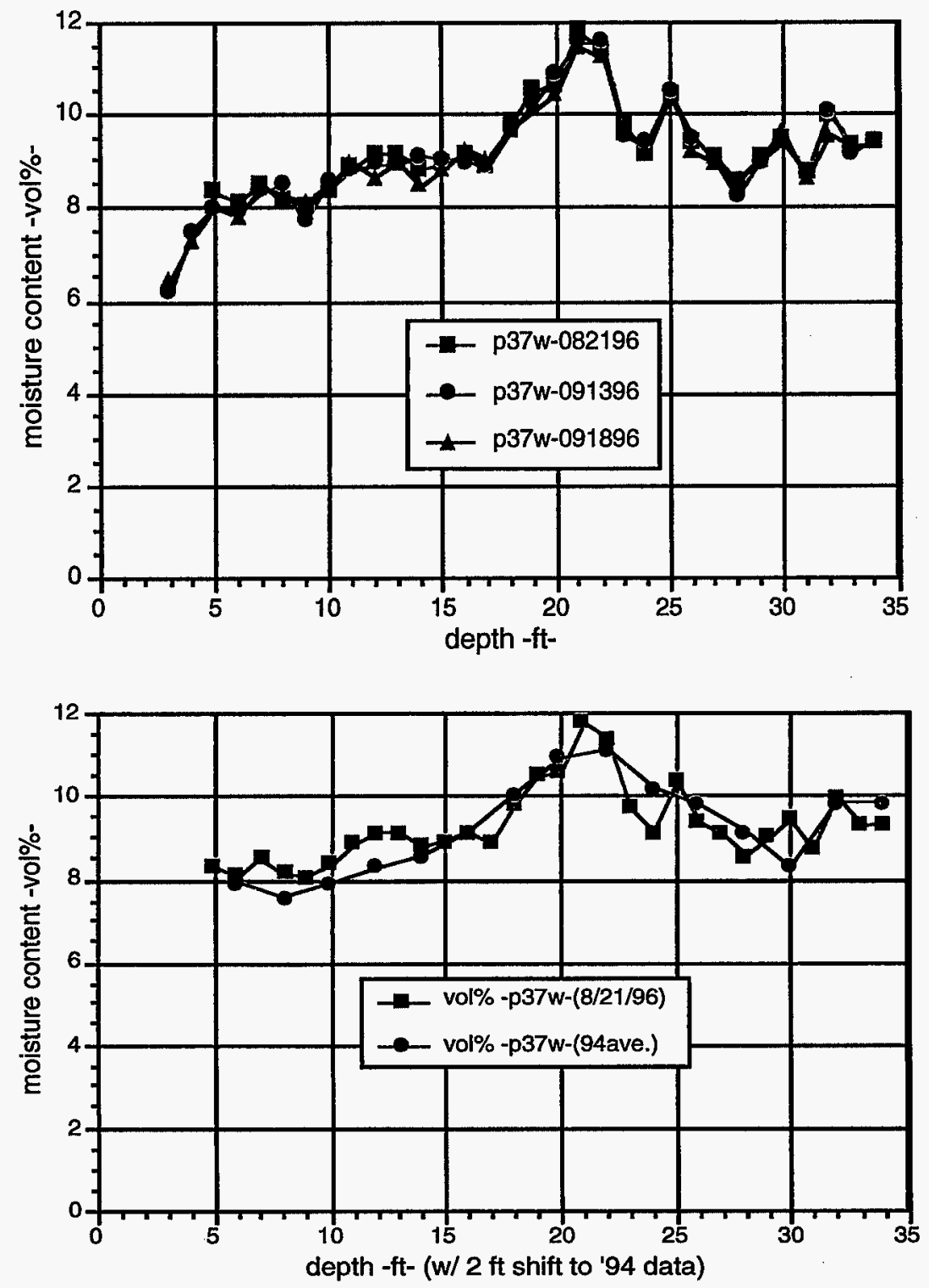

Fig. 21 Moisture profiles in pit 37 (west). Good reproducibility of profile features is evident in the three profiles (top), taken in 1996. The August 1996 profile is in good agreement with the average profile from 1994 (94ave) in the (bottom) figure. 


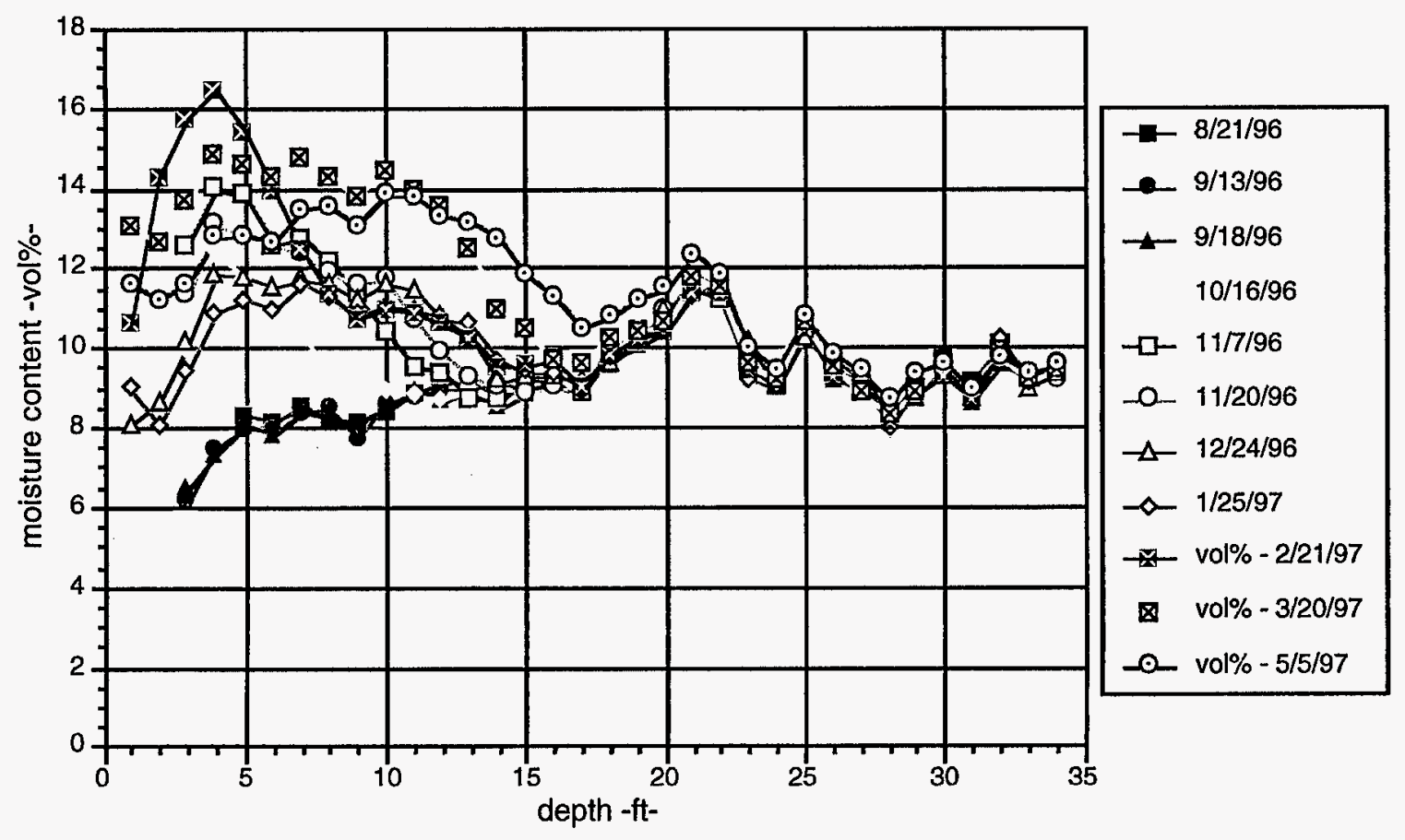

Fig. 22 Moisture profiles in pit 37 (west) taken from August 1996 to May 1997 show a significant pulse of moisture moving through the profile. 

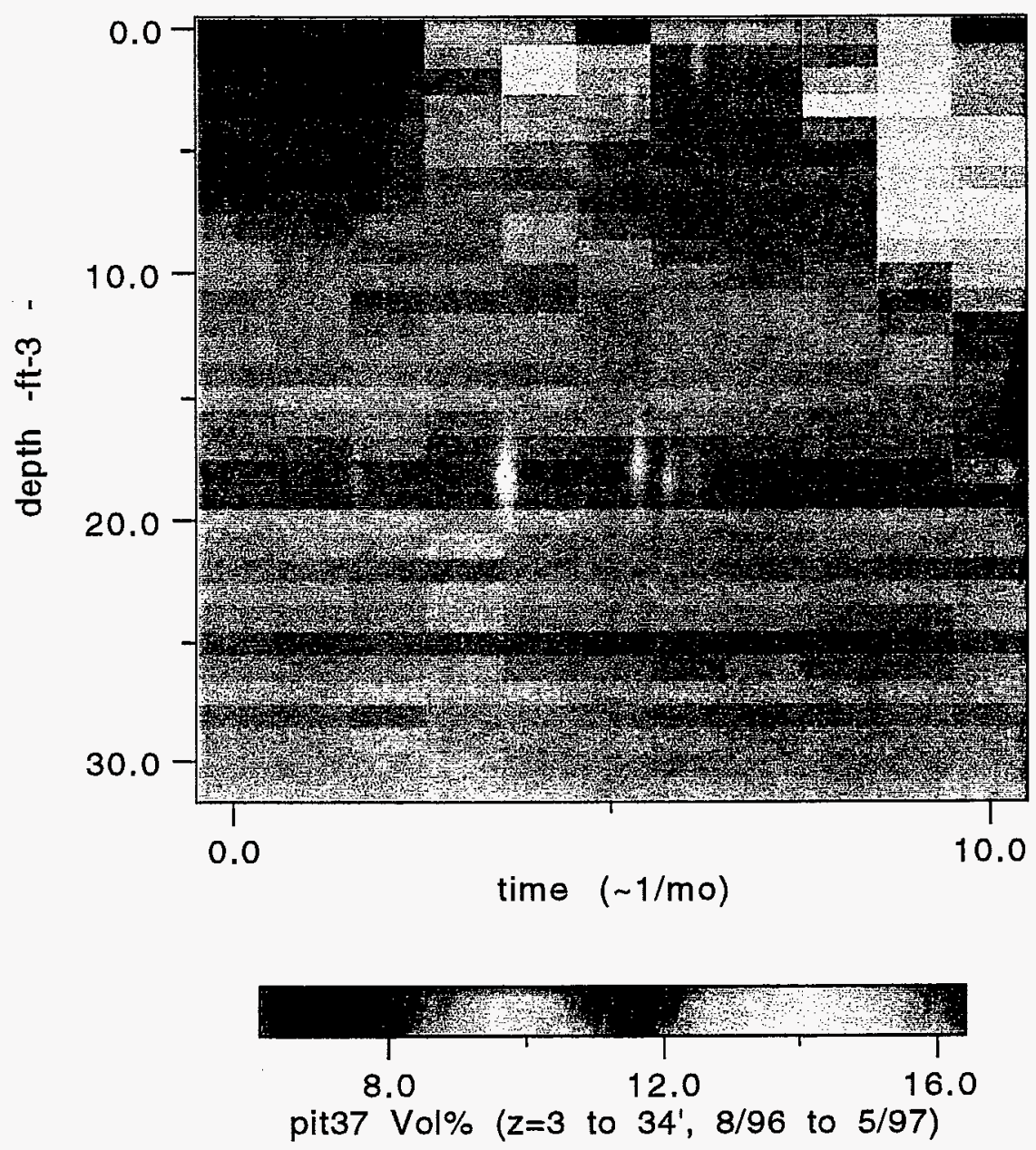
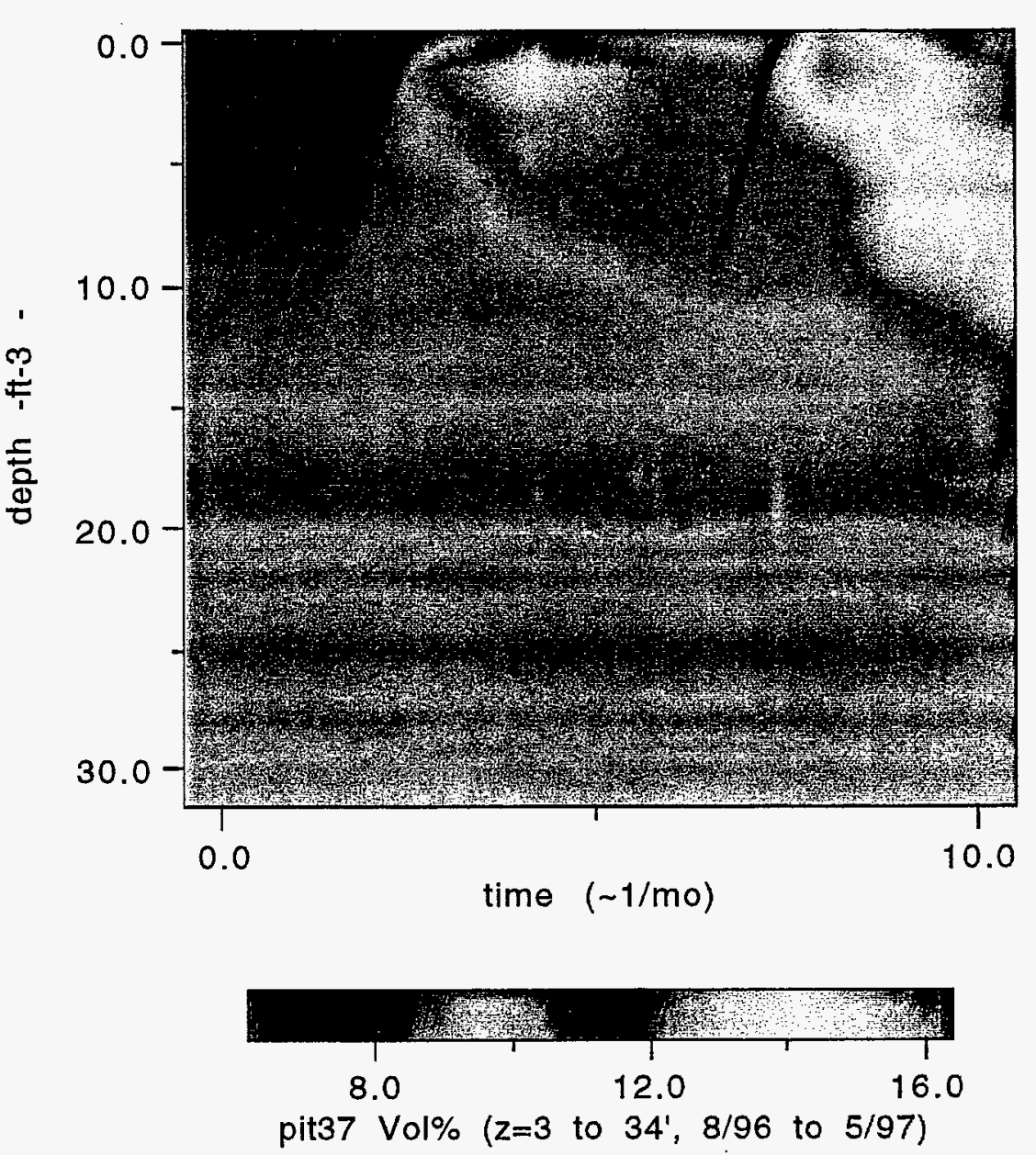

Fig. 23 Moisture content verses depth and time over approximately one year (8/95 to 6/96). The left figure shows the actual resolution of the neutron moisture probe and the right figure shows a smooth interpolation of the data. The two moisture pulses seen near the surface start in the fall (following summer rains) and in the spring (following snow melt), with the snow melt moisture pulse being more significant in this case. 

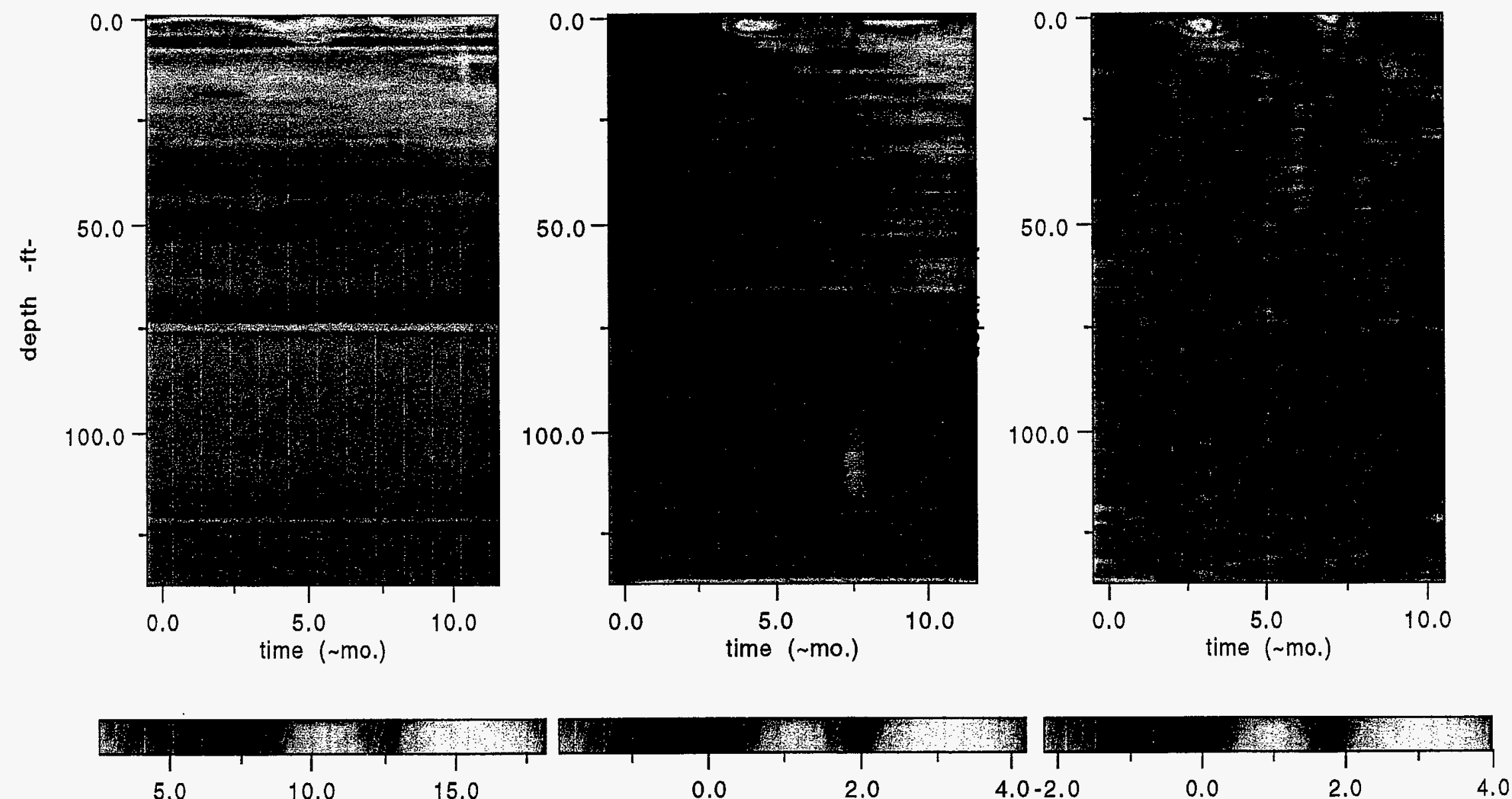

moisture content in 1121 -vol\%- moisture change in 1121 since 9/18/96 -vol\%tunning change in 1121 moisture -vol\%-

Fig. 24 Moisture content (left) in vol\% in borehole 1121 shown verses depth (ft) and time ( monthly), with moisture change since the first profile (center) and moisture difference since the previous months profile (right). 

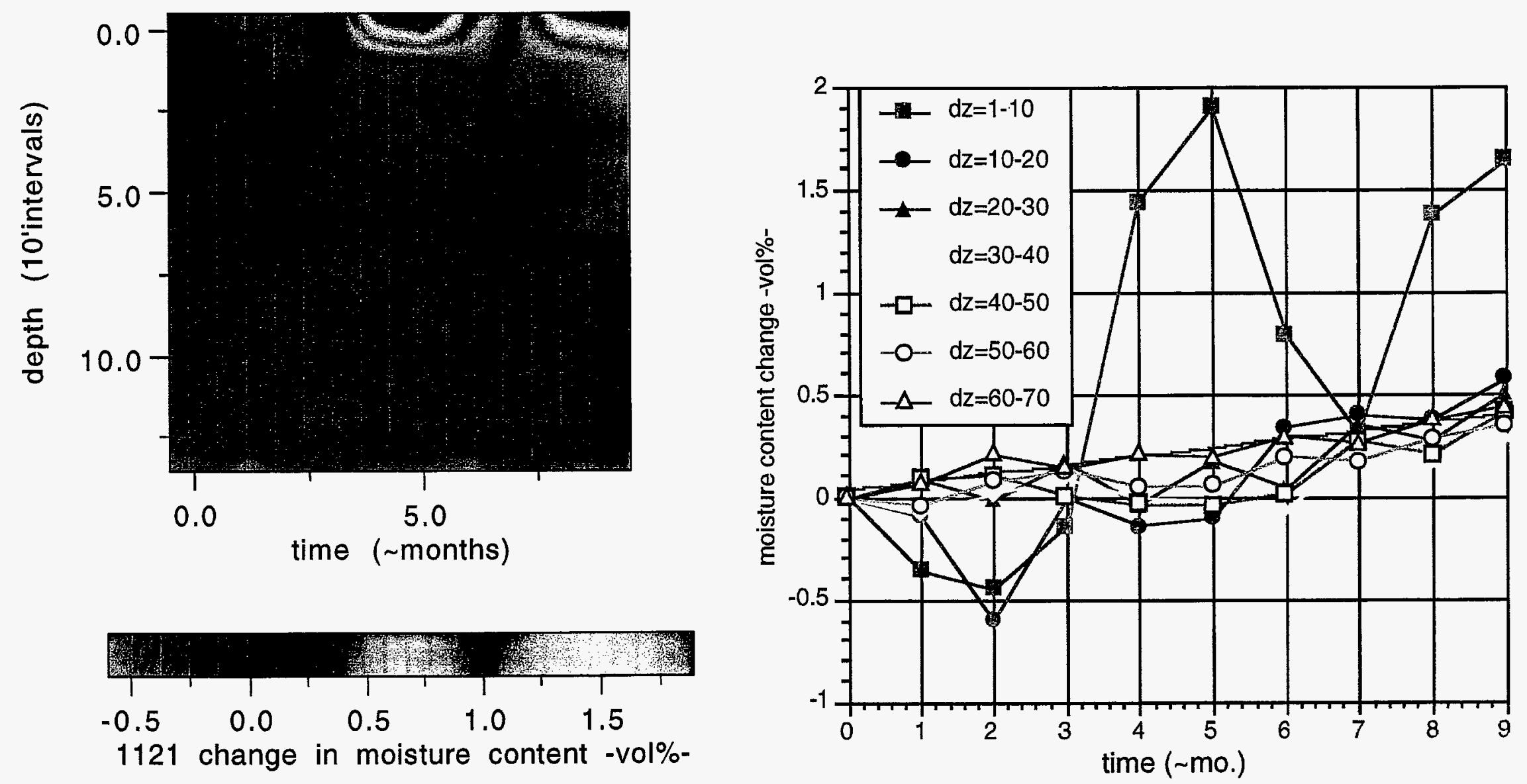

Fig. 25 (Left) Change in moisture content since the first profile (vol\%) for borehole 1121 for moisture contents averaged over each $10^{\prime}$ depth interval, shown verses depth interval and time ( monthly). (Right) Same data is plotted verses time for the uppermost seven $10^{\prime}$ depth intervals. 


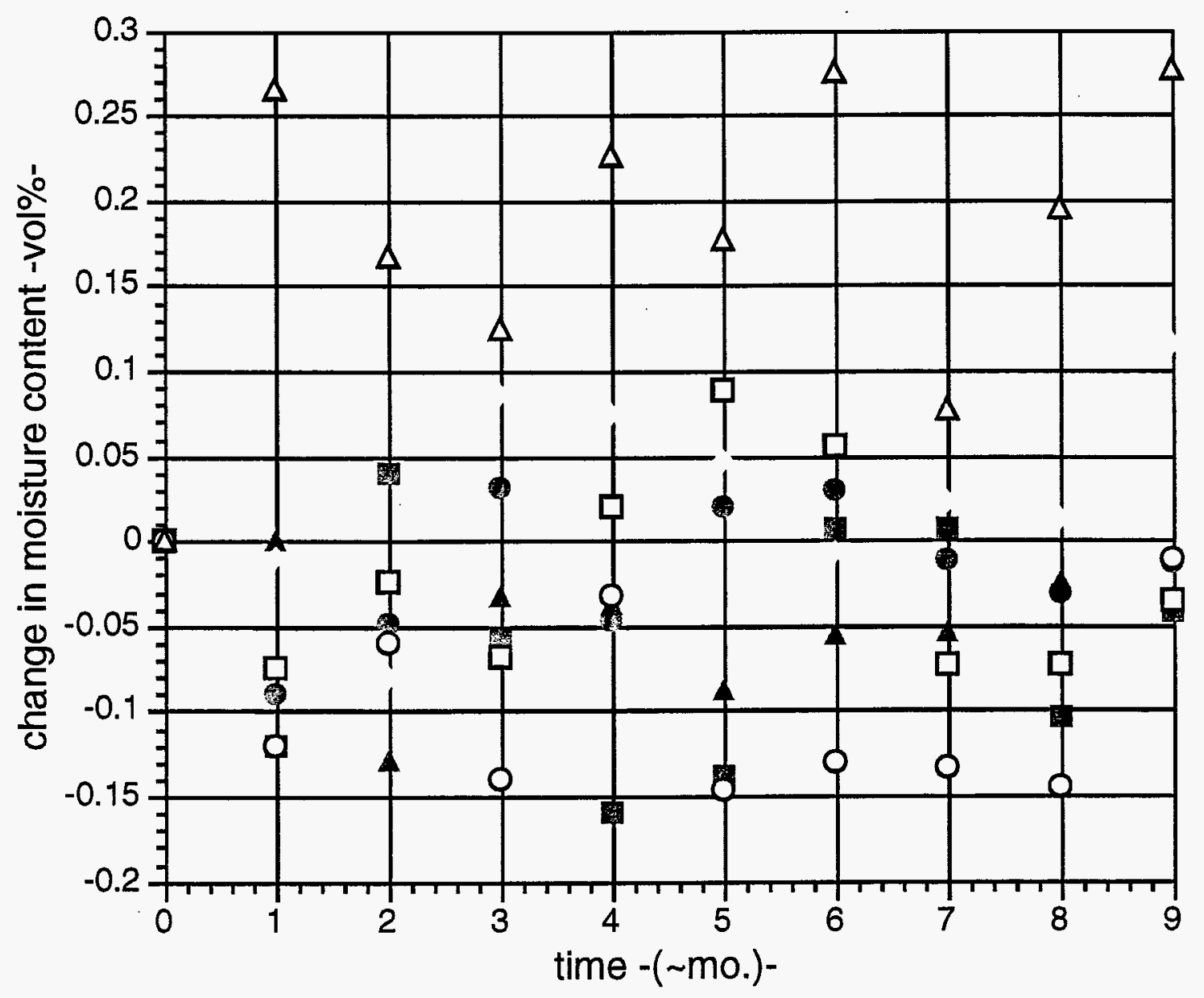

$\mathrm{dz}=70-80$

$\mathrm{dz}=80-90$

A $\mathrm{dz}=90-100$ $d z=100-110$

口 $\mathrm{dz}=110-120$

O $d z=120-130$

$\Delta d z=130-140$

Fig. 26 Change in moisture content since the first profile (vol\%) for borehole 1121 plotted verses time for the deepest seven 10' depth intervals (see Fig.24). 

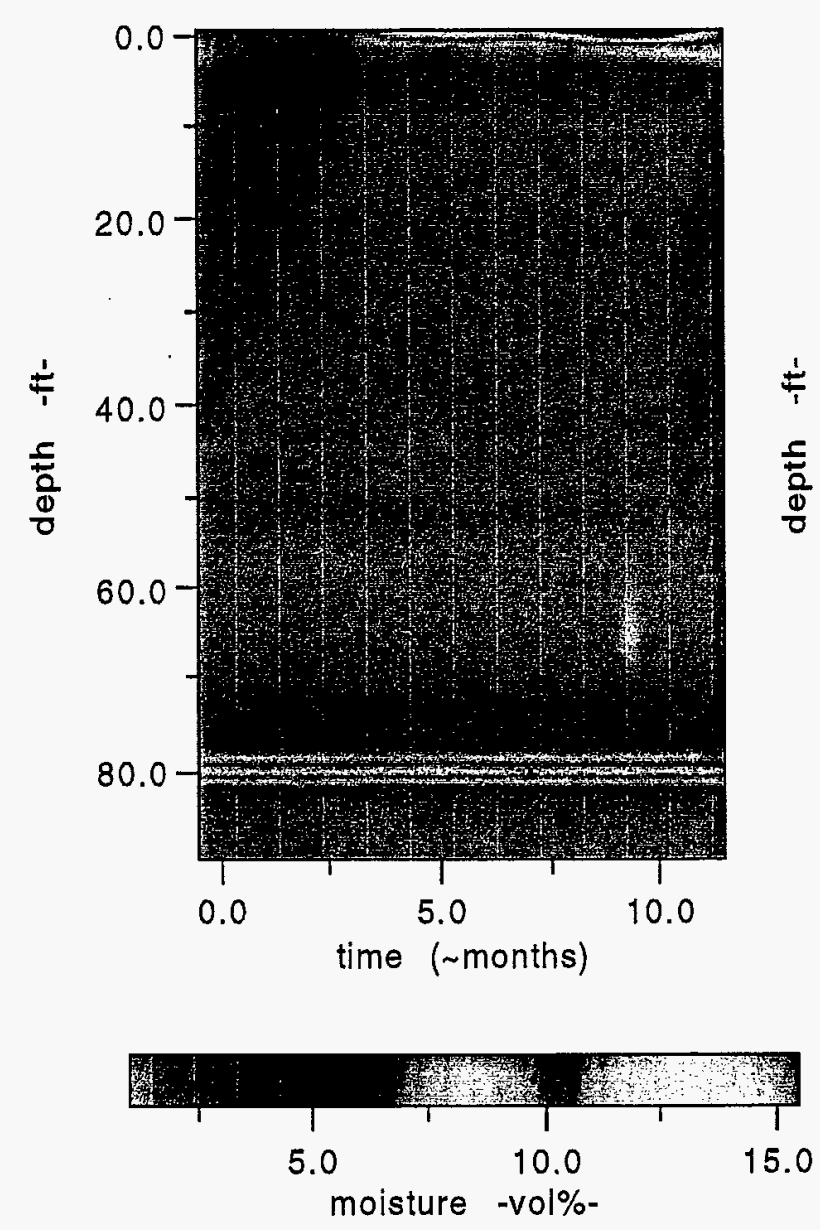
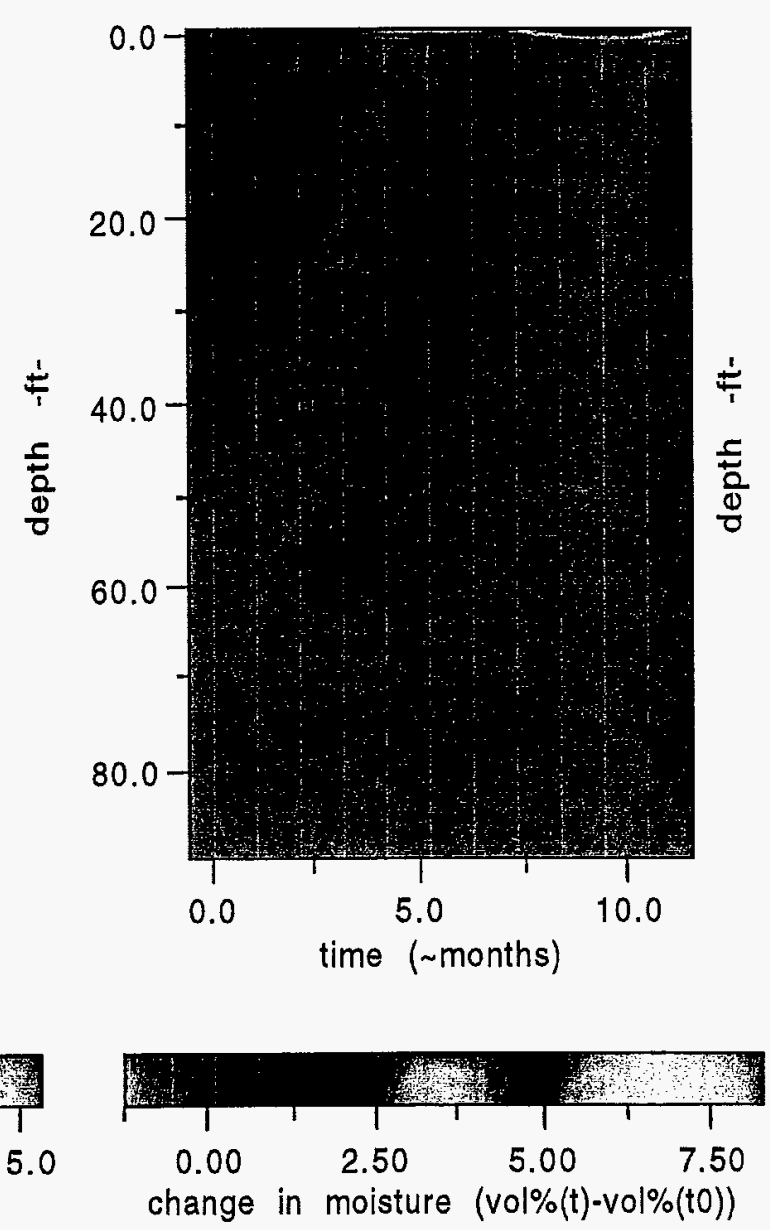
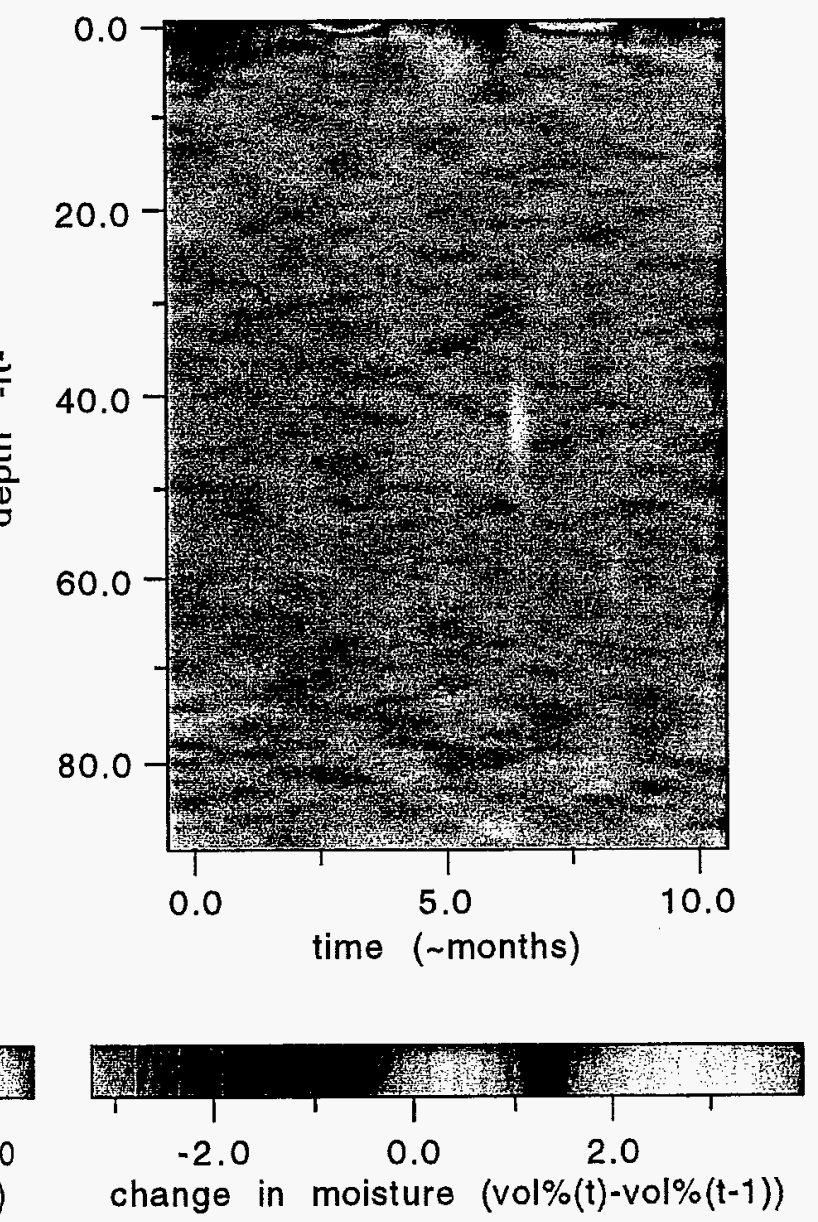

Fig. 27 Moisture content (left) in vol\% in borehole 1117 shown verses depth (ft) and time ( monthly), with moisture change since the first profile (center) and moisture difference since the previous months profile (right). 


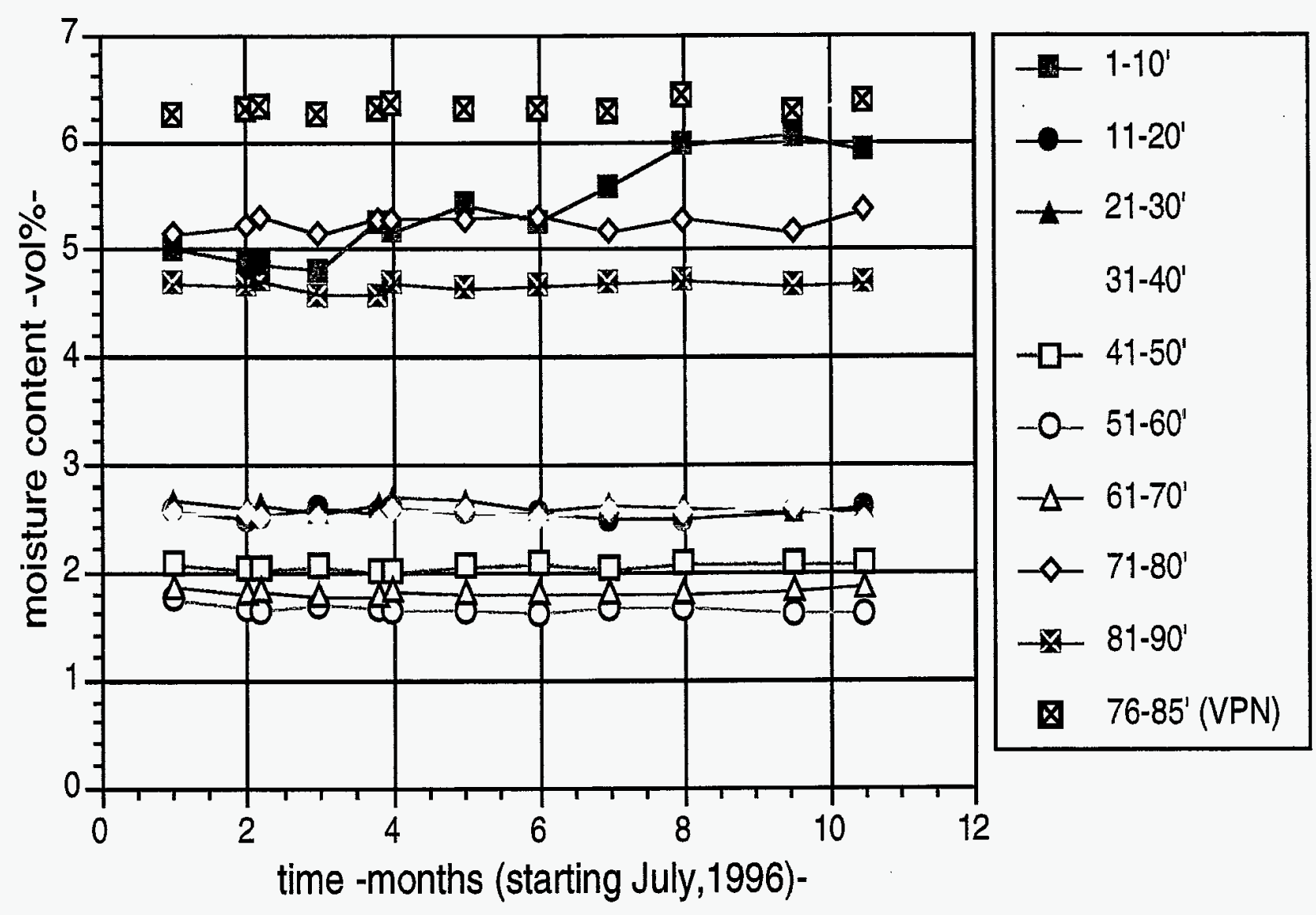

Fig. 28 Change in moisture content since the first profile (vol\%) for borehole 1117 plotted verses time for the nine $10^{\prime}$ depth intervals (see Fig.26) in the borehole and the interval (76-85') including the vapor phase notch (VPN) region. 

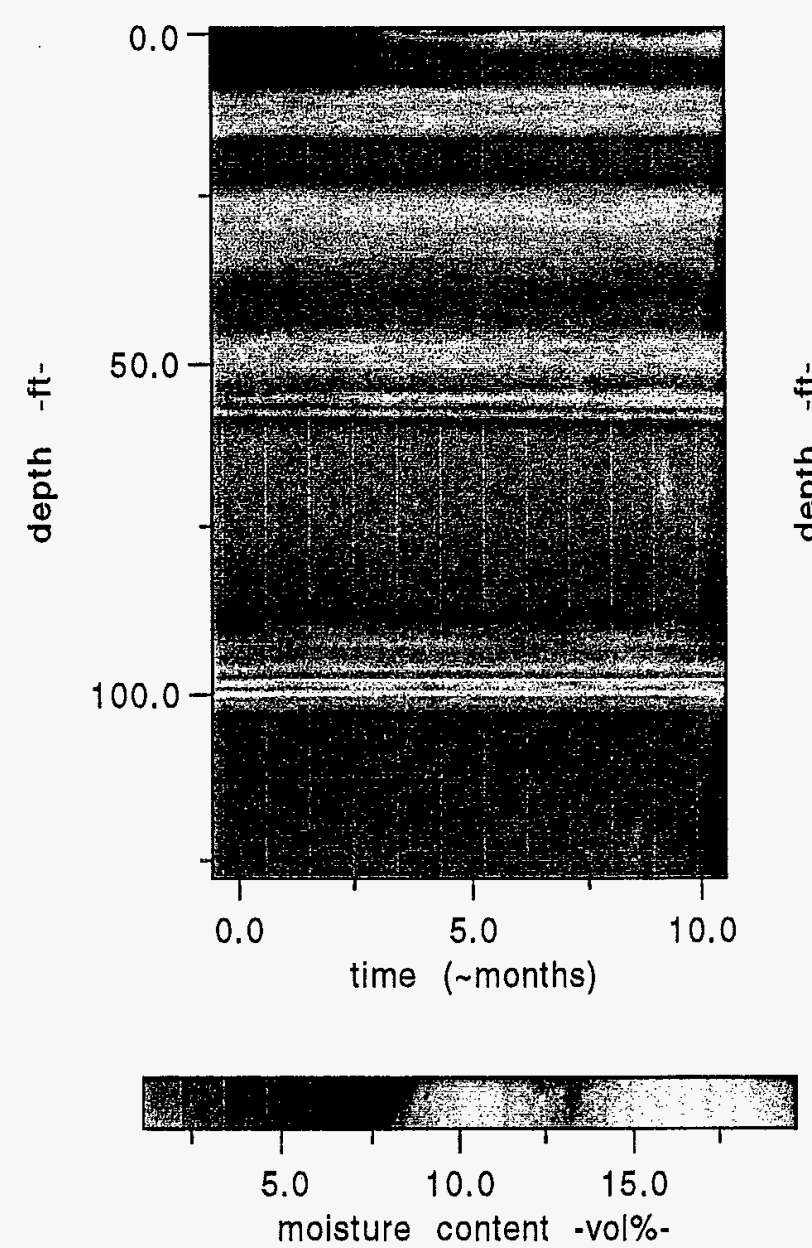
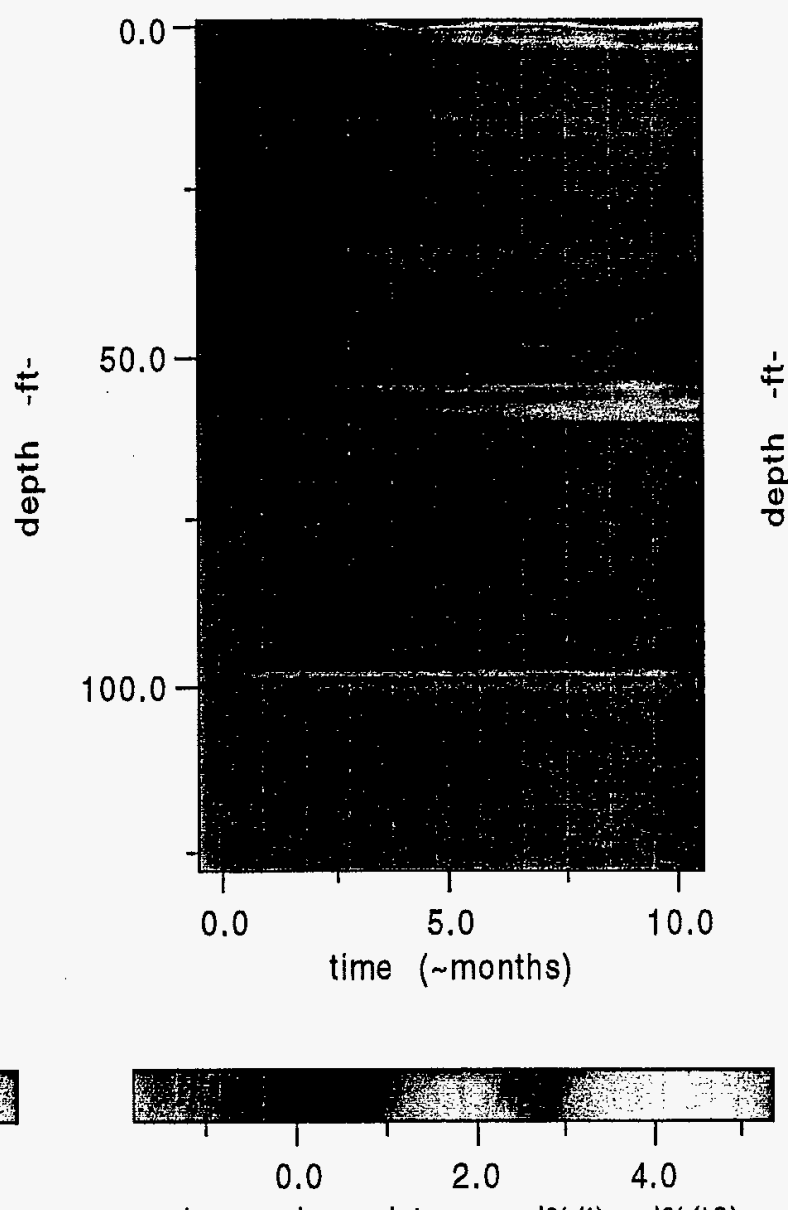

change in moisture: vol\%(t)-vol\%(t0)
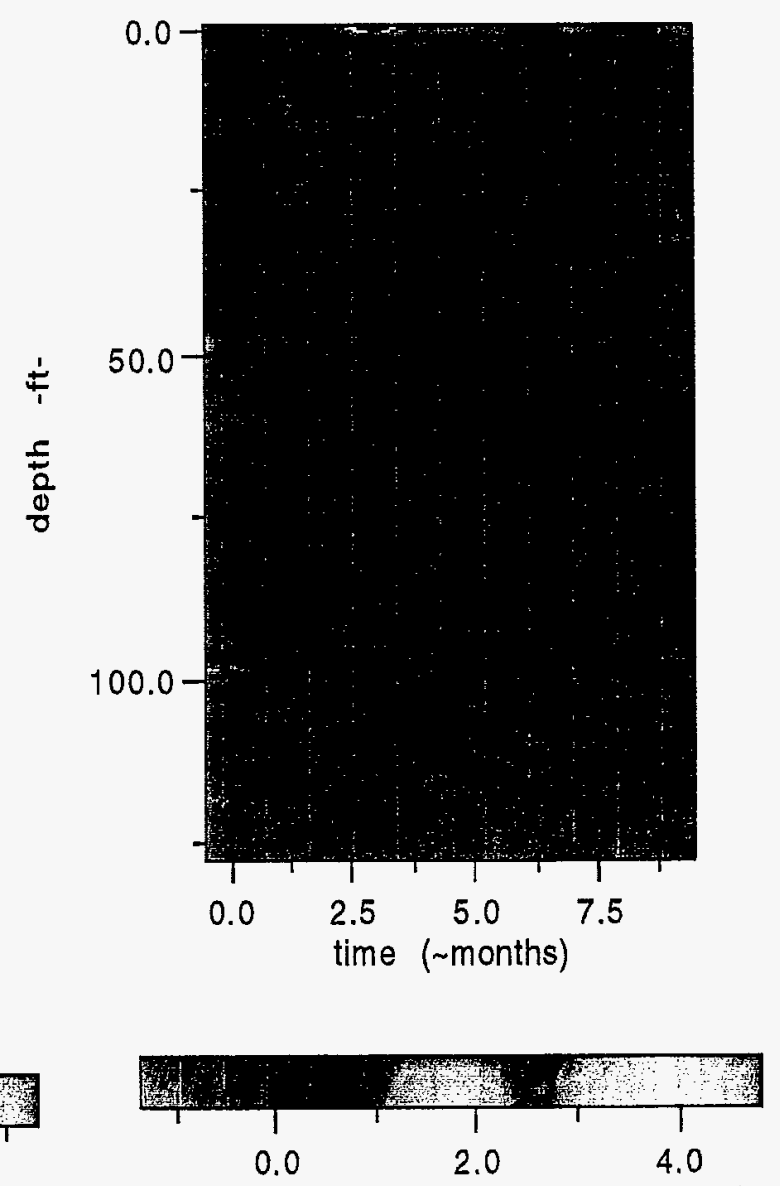

change in moisture (vol\%(t)-vol\%(t-1))

Fig. 29 Moisture content (left) in vol\% in borehole 1107 shown verses depth (ft) and time ( $\sim$ monthly), with moisture change since the first profile (center) and moisture difference since the previous months profile (right). 

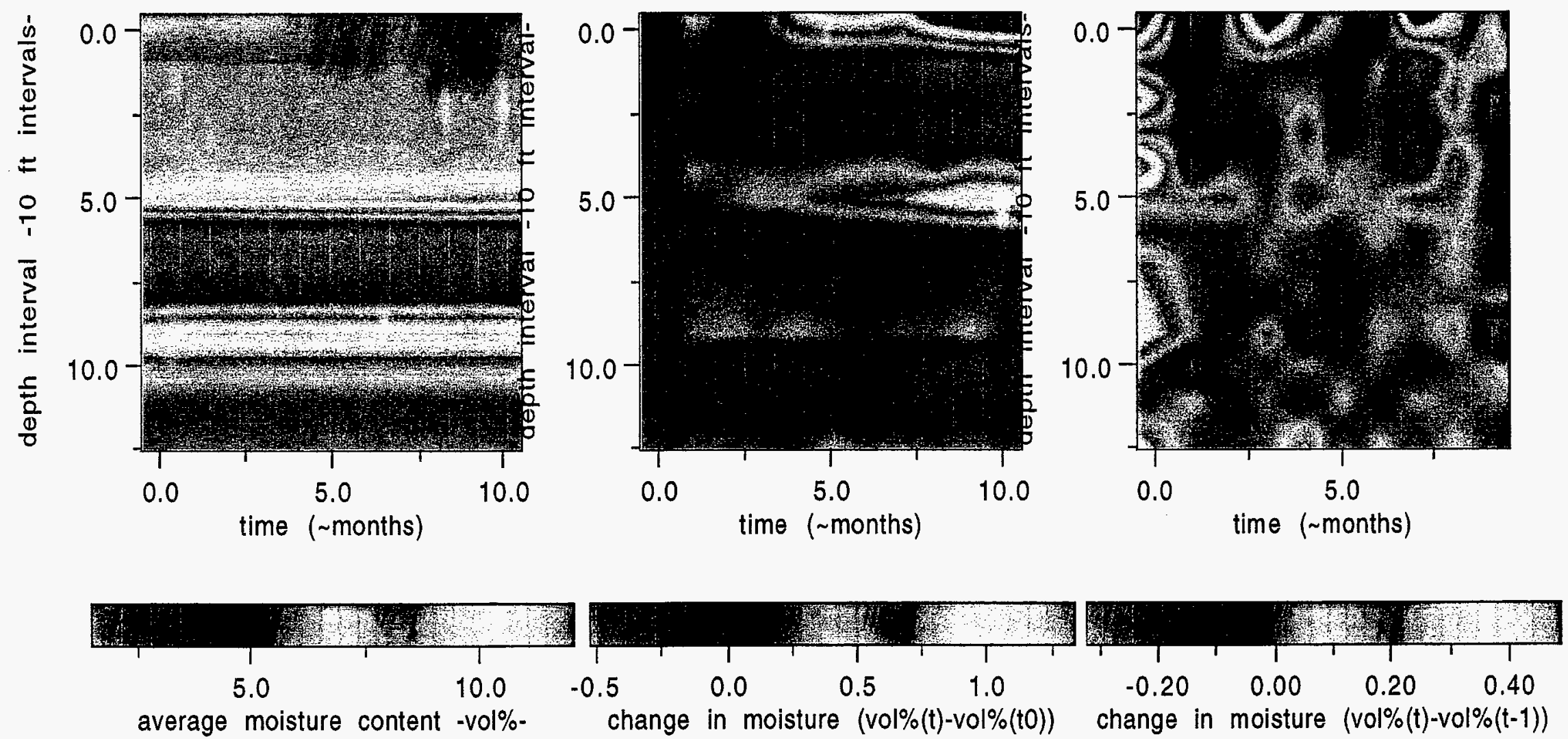

Fig. 30 Moisture content (left) in vol\% in borehole 1107 averaged over $10^{\prime}$ depth intervals shown verses $10^{\prime}$ depth interval and time ( monthly), with similar plots for moisture change since the first profile (center) and moisture difference since the previous months profile (right). 


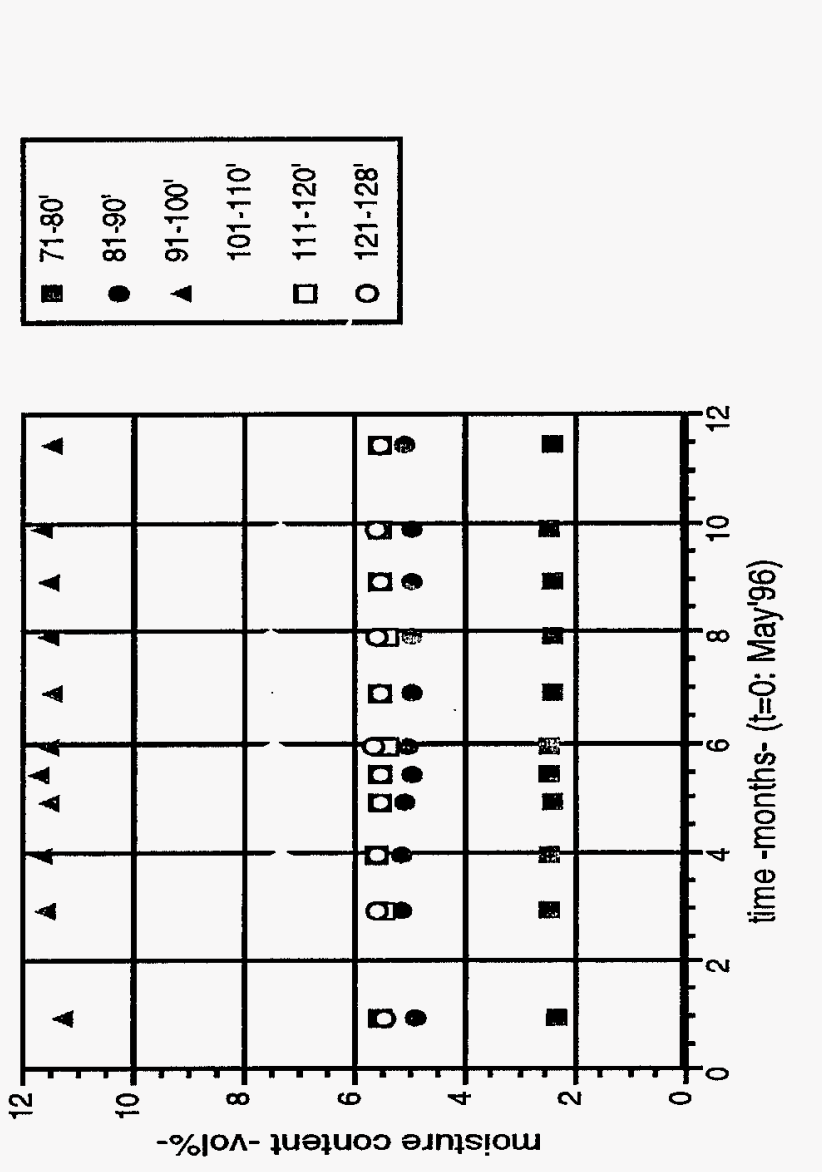

䄵

는

导

D

$>$

T

芒

옹

0
0
$\frac{1}{4}$
$\frac{0}{0}$
0

हैํำ

$\frac{2}{2}$

현

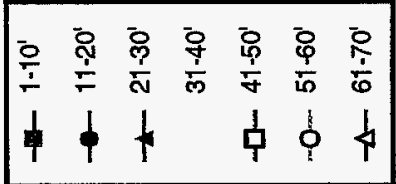

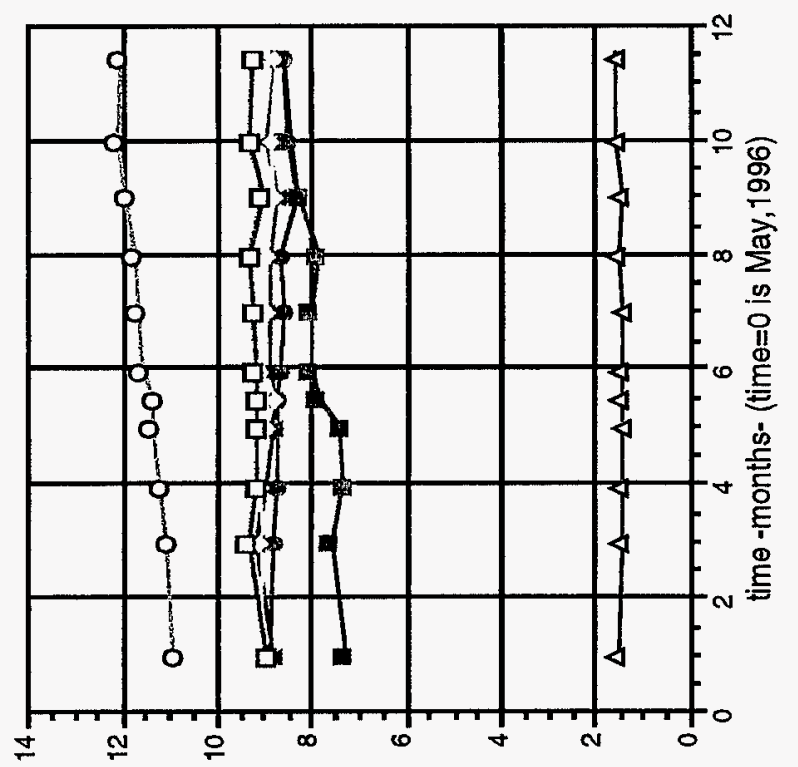

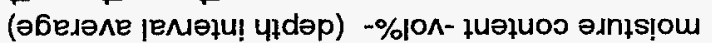

숭

$+\frac{1}{0}$

语

옹

甲

皇.马

+ จิ

声

엉

过

車

늉

द्व के

ક.

o 兵

인은

穵

त)

我. 


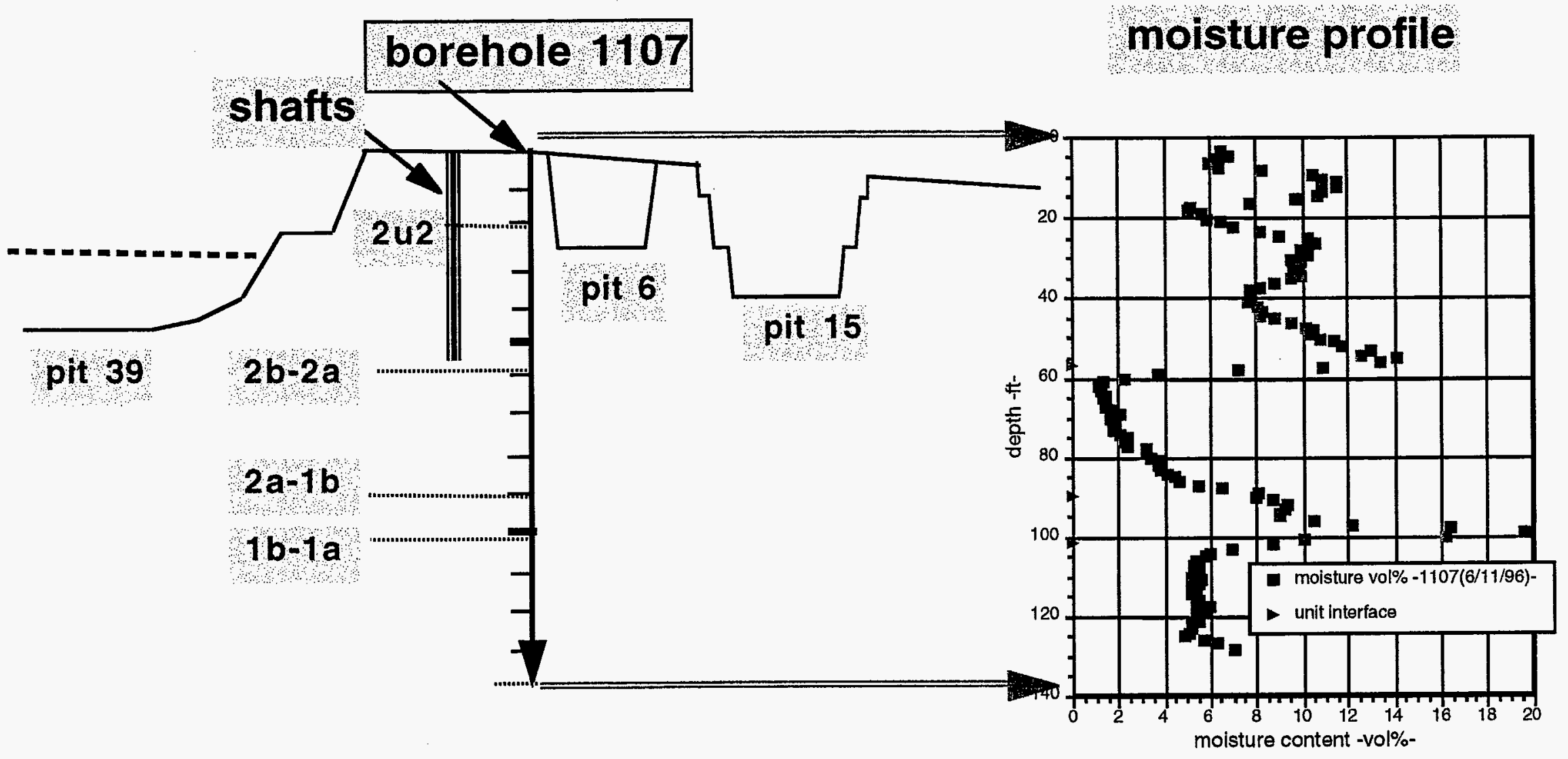

Fig. 32 Area G horizon profiles near borehole 1107. 


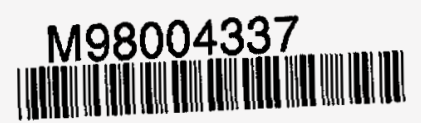

Report Number (14) $L A=\chi R=-97-5186$

Publ. Date (11)

Sponsor Code (18) $\frac{D S E / E M, X F}{U C-2000, D O E / E R}$
uC Category (19) 